\title{
Intrinsic interference mitigating coordinated beamforming for the FBMC/OQAM based downlink
}

\author{
Yao Cheng ${ }^{*}$, Peng Li and Martin Haardt
}

\begin{abstract}
In this work, we propose intrinsic interference mitigating coordinated beamforming (IIM-CBF)-based transmission strategies for the downlink of multi-user multiple-input-multiple-out (MIMO) systems and coordinated multi-point (COMP) systems where filter bank based multi-carrier with offset quadrature amplitude modulation (FBMC/OQAM) is employed. Our goal is to alleviate the dimensionality constraint imposed on the state-of-the-art solutions for FBMC/OQAM-based space division multiple access that the total number of receive antennas of the users must not exceed the number of transmit antennas at the base station. First, two IIM-CBF algorithms are developed for a single-cell multi-user MIMO downlink system. The central idea is to jointly and iteratively calculate the precoding matrix and decoding matrix for each subcarrier to mitigate the multi-user interference as well as the intrinsic interference inherent in FBMC/OQAM-based systems. Second, for a COMP downlink scenario where partial coordination among the base stations is considered, the application of coordinated beamforming-based transmission schemes is further investigated. An appropriate IIM-CBF technique is proposed. Simulation results show that when the number of transmit antennas at the base station is equal to the total number of receive antennas of the users, the proposed IIM-CBF algorithm outperforms the existing transmission strategies for FBMC/OQAM-based multi-user MIMO downlink systems. Moreover, we evaluate the performances of the IIM-CBF schemes in the downlink of multi-user MIMO systems and CoMP systems where the total number of receive antennas of users exceeds the number of transmit antennas at the base station. It is observed that by employing the IIM-CBF techniques, FBMC/OQAM systems achieve a similar bit error rate (BER) performance as its orthogonal frequency division multiplexing with the cyclic prefix insertion (CP-OFDM)-based counterpart while exhibiting superiority in terms of a higher spectral efficiency, a greater robustness against synchronization errors, and a lower out-of-band radiation. In the presence of residual carrier frequency offsets, FBMC/OQAM systems provide a much better performance compared to the CP-OFDM-based system, which corroborates the theoretical analysis that FBMC/OQAM systems are more immune to the lack of perfect synchronization. In addition, numerical results with respect to the convergence behavior of the IIM-CBF techniques are presented, and the computational complexity issue is also addressed.
\end{abstract}

\section{Introduction}

As a promising alternative to orthogonal frequency division multiplexing with the cyclic prefix insertion (CPOFDM), filter bank based multi-carrier modulation (FB$\mathrm{MC})$ has received great research attention in recent years. Using spectrally well-contained synthesis and analysis of filter banks at the transmitter and at the receiver [1,2], FB-MC has an agile spectrum. Thereby, the out-of-band

*Correspondence: y.cheng@tu-ilmenau.de

Communications Research Laboratory, Ilmenau University of Technology, P.O. Box 100565, IImenau 98684, Germany radiation is lower compared to CP-OFDM, and it is consequently beneficial to choose FB-MC over CP-OFDM for asynchronous scenarios [3,4] or to achieve an effective utilization of spectrum holes [5,6]. Moreover, in systems where filter bank based multi-carrier with offset quadrature amplitude modulation (FBMC/OQAM) is employed, the fact that the insertion of the $\mathrm{CP}$ is not required as in CP-OFDM-based systems leads to a higher spectral efficiency.

In FBMC/OQAM systems, the real and imaginary parts of each complex-valued data symbol are staggered by half

\section{黑 Springer}

C) 2014 Cheng et al: licensee Springer. This is an Open Access article distributed under the terms of the Creative Commons Attribution License (http://creativecommons.org/licenses/by/2.0), which permits unrestricted use, distribution, and reproduction in any medium, provided the original work is properly credited. 
of the symbol period $[2,7]$ such that the desired signal and the intrinsic interference are separated in the real domain and in the pure imaginary domain, respectively. Different approaches of canceling the intrinsic interference have been proposed based on different assumptions on the frequency selectivity of the channel. In [8] and [9] where receive processing techniques have been developed for multiple-input-multiple-out (MIMO) FBMC/OQAM systems, it is assumed that the channel frequency responses of adjacent subcarriers do not vary. Consequently, the intrinsic interference is canceled by taking the real part of the resulting signal after the equalization.

To alleviate the constraint on the frequency selectivity of the channel, a zero forcing (ZF)-based approach has been proposed in [10] for multi-stream transmissions in a MIMO FBMC/OQAM system where the channel is not restricted to flat fading. More details of the performance analysis of this algorithm have been presented in [11]. However, the work in [10] and [11] is limited to the case where the number of receive antennas does not exceed the number of transmit antennas. In addition, the authors have shown numerically and have also pointed out that their proposed approach only provides a satisfactory performance in an asymmetric configuration, i.e., when the number of transmit antennas is larger than the number of receive antennas. Based on the concept of mitigating the intrinsic interference mentioned above for point-to-point MIMO FBMC/OQAM systems, the authors in [12] have adapted the conventional spatial Tomlinson-Harashima precoder (STHP) to an FBMC/OQAM-based multipleinput-single-output broadcast channel (MISO-BC) which results in a new nonlinear precoder. It is known that nonlinear precoders have a higher computational complexity compared to linear precoders. Moreover, the nonlinear precoding technique in [12] is restricted to the case where each user is equipped with only a single receive antenna. On the other hand, a block diagonalization (BD)based linear precoder has been developed in [13] for the FBMC/OQAM-based multi-user MIMO downlink with space division multiple access (SDMA). It adopts the central idea of $\mathrm{BD}$ [14] to mitigate the multi-user interference and then uses the ZF-based approach [10] to deal with the intrinsic interference cancelation for the resulting equivalent single-user transmissions. Consequently, this algorithm inherits the drawback of the ZF-based scheme such that it also fails to achieve a good performance in a symmetric multi-user MIMO downlink setting, where the number of transmit antennas at the base station is equal to the total number of receive antennas of the users. In addition, this linear precoder suffers from the dimensionality constraint that the total number of receive antennas of the users must not exceed the number of transmit antennas at the base station. Note that similarly as in these publications, we focus on scenarios where the channel on each subcarrier is flat fading in this work.

Although the case of highly frequency selective channels is beyond the scope of this paper, we also refer to two techniques designed for such scenarios to complete the review of the state-of-the-art transmission schemes for FBMC/OQAM-based multi-user downlink systems. The linear precoder in [15] has a structure of a filter applied on each subcarrier and its two adjacent subcarriers at twice the symbol rate. It also only focuses on the setting where the number of transmit antennas at the base station is not smaller than the total number of receive antennas of the users. Moreover, it only allows each user to have a single receive antenna, and consequently only one data stream can be transmitted to each user. In [16], transmission schemes for FBMC/OQAM-based multi-user MISO downlink systems have been developed also considering highly frequency selective channels. The authors have devised two different minimum mean square error (MMSE) approaches. A close-form solution is provided in the first one, while the second scheme involves a joint transmitter and receiver design via an iterative procedure. Note that in the aforementioned publications on the FBMC/OQAM-based multi-user downlink, the impact of the residual carrier frequency offsets (CFOs) has not been investigated.

To the best of the authors' knowledge, FBMC/OQAMbased coordinated multi-point (CoMP) techniques have not been studied in the literature. In the context of CPOFDM, the corresponding research has been very fruitful, and CoMP is known as one of the advanced communication techniques that are able to provide benefits of reduced inter-cell interference and enhanced cell edge throughput [17-21]. In this work, we focus on downlink CoMP and the schemes that belong to the category of joint transmission [18]. When the full cooperation between the base stations of adjacent cells is assumed, the channel state information (CSI) and signals for all users are shared by the base stations. In this case, a virtual multi-user MIMO downlink setting is formed, where the transmit antennas are geographically separated. Thereby, the transmission strategies that have been developed for the single-cell multi-user MIMO downlink can be employed. Nevertheless, such a full cooperation scheme is not practical due to issues such as it requires excessive information exchange resulting in a large signaling overhead, and the CSI of all users is very hard to acquire [19]. As a more realistic solution, partial cooperation schemes have been proposed in $[19,21,22]$, where the users are classified into two categories, cell (or in some papers [19,21], cluster that consists of multiple cells) interior users and cell (cluster) edge users. The base stations of adjacent cells (clusters) transmit the same signals to the cell (cluster) edge users and coordinate beamforming techniques that 
rely on the limited cooperation between the cells (clusters) (e.g., the exchange of the beamforming matrices for cell (cluster) edge users) are employed to suppress the intra-cell (cluster) and inter-cell (cluster) interference. For these downlink CoMP scenarios, it is more likely that the total number of receive antennas of the users served by one base station is larger than the number of transmit antennas. Thus, transmission strategies that are able to tackle such a case are required. Note that in the aforementioned publications on CP-OFDM-based downlink CoMP, perfect synchronization is assumed. However, the asynchronous nature of the interference in the downlink CoMP setting is emphasized in [23]. It has been shown in [23] that the lack of perfect synchronization causes a performance degradation. Such a fact greatly motivates the use of FB-MC as a replacement of CP-OFDM, as FB-MC is more robust against synchronization errors compared to CP-OFDM.

In this paper, we design intrinsic interference mitigating coordinated beamforming (IIM-CBF) schemes ${ }^{\mathrm{a}}$ for the FBMC/OQAM-based multi-user MIMO downlink systems and CoMP downlink systems without restricting the configuration with respect to the number of transmit antennas and the number of receive antennas. First, considering the symmetric single-cell multi-user MIMO downlink setting where the number of transmit antennas at the base station is equal to the total number of receive antennas of the users, we propose to compute the precoding matrix and the decoding matrix jointly in an iterative procedure for each subcarrier. Different choices of the decoding matrix in the initialization step are recommended for different scenarios. For CP-OFDM-based multi-user MIMO downlink systems, there have been some publications on coordinated beamforming techniques $[19,24]$ proposed to cope with the dimensionality constraint imposed on BD-based precoding algorithms [14]. Inspired by these works, an IIM-CBF scheme specifically for FBMC/OQAM-based systems is developed to alleviate the same dimensionality constraint that all state-of-the-art transmission strategies for FBMC/OQAM-based multi-user downlink settings suffer from. It handles the mitigation of the multi-user interference as well as the intrinsic interference. Moreover, we investigate FBMC/OQAM-based CoMP techniques for the first time and provide an extension of the IIM-CBF scheme designed for the FBMC/OQAMbased multi-user MIMO downlink system. To evaluate the performance of the proposed IIM-CBF algorithms, we have performed extensive simulations. The bit error rate (BER) as well as sum rate performances are shown, and the convergence behavior of the developed coordinated beamforming techniques is also investigated via numerical simulations. It can be observed that the number of iterations required for the convergence in these
IIM-CBF schemes is acceptable. Thus, the additional computational complexity is not prohibitive compared to the close-form algorithms which fail in scenarios where the total number of receive antennas of the users exceeds the number of transmit antennas. In addition, we also investigate the effects of the residual CFOs and demonstrate the superiority of the FBMC/OQAM-based system over its CP-OFDM-based counterpart in the tolerance of synchronization errors.

The remainder of the paper is organized as follows: Section 2 introduces the data model of a single-cell multiuser MIMO FBMC/OQAM system and reviews two stateof-the-art transmission strategies for such a system. In Section 3, the two proposed algorithms IIM-CBF 1 and IIM-CBF 2 are described in detail for the case where the number of transmit antennas at the base station is equal to the total number of receive antennas of the users and the case where the former is smaller than the latter, respectively. Section 4 focuses on the CoMP downlink and presents another coordinated beamforming-based transmission scheme, namely 'IIM-CBF 3'. Numerical results are shown in Section 5, before conclusions are drawn in Section 6.

Notation Matrices and vectors are denoted by boldfaced uppercase and lowercase letters, respectively. We use the superscripts ${ }^{\mathrm{T}},{ }^{\mathrm{H}}$, and ${ }^{-1}$ for transpose, Hermitian transpose, and matrix inversion, respectively. An $M \times M$ identity matrix is denoted by $\boldsymbol{I}_{M}$. The Frobenius norm of a matrix is denoted by $\|\cdot\|_{\mathrm{F}}$. Moreover, $\operatorname{Re}\{\cdot\}$ symbolizes the real part of the input argument, while $\operatorname{Im}\{\cdot\}$ represents the imaginary part. For a matrix $\boldsymbol{A}, \boldsymbol{A}(m, n)$ denotes its $(m, n)$ th entry.

\section{System model}

In a multi-user MIMO downlink system where SDMA is employed, one base station equipped with $M_{\mathrm{T}}^{(\mathrm{BS})}$ transmit antennas transmits to $Q$ users at the same time and on the same frequency. The number of receive antennas of the $q$ th user is denoted by $M_{\mathrm{R}_{q}}$, and the total number of receive antennas of all users severed simultaneously is then $M_{\mathrm{R}}^{\text {(tot) }}=\sum_{q=1}^{Q} M_{\mathrm{R}_{q}}$. Assuming that the channel on each subcarrier can be treated as flat fading $[10,11,13]$, the combined receive vector on the $k$ th subcarrier and at the $n$th time instant is denoted by $\boldsymbol{y}_{k}[n]=\left[\boldsymbol{y}_{1, k}^{\mathrm{T}}[n] \boldsymbol{y}_{2, k}^{\mathrm{T}}[n] \cdots \boldsymbol{y}_{Q, k}^{\mathrm{T}}[n]\right]^{\mathrm{T}} \in \mathbb{C}^{M_{\mathrm{R}}^{(\text {(tot) }}}$ where the received signals of all $Q$ users are stacked and can be represented by

$$
\begin{aligned}
\boldsymbol{y}_{k}[n]= & \boldsymbol{H}_{k}[n] \boldsymbol{F}_{k}[n] \boldsymbol{d}_{k}[n]+\sum_{i=n-3}^{n+3} \sum_{\ell=k-1}^{k+1} \boldsymbol{H}_{\ell}[i] \boldsymbol{F}_{\ell}[i] c_{i \ell} \boldsymbol{d}_{\ell}[i] \\
& +\boldsymbol{n}_{k}[n], \quad(\ell, i) \neq(k, n) .
\end{aligned}
$$


Here $\boldsymbol{H}_{k}[n] \in \mathbb{C}^{M_{\mathrm{R}}^{(\mathrm{tot})} \times M_{\mathrm{T}}^{(\mathrm{BS})}}$ denotes the combined channel matrix of all $Q$ users $^{\mathrm{b}}$ and is written as

$$
\boldsymbol{H}_{k}[n]=\left[\boldsymbol{H}_{1, k}^{\mathrm{T}}[n] \boldsymbol{H}_{2, k}^{\mathrm{T}}[n] \cdots \boldsymbol{H}_{Q, k}^{\mathrm{T}}[n]\right]^{\mathrm{T}},
$$

where $\boldsymbol{H}_{q, k}[n] \in \mathbb{C}^{M_{\mathrm{R}_{q}} \times M_{\mathrm{T}}^{(\mathrm{BS})}}$ represents the channel frequency response between the base station and the $q$ th user, $q=1,2, \ldots, Q$. The data vector $\boldsymbol{d}_{k}[n] \in \mathbb{R}^{d}$ with the total number of spatial streams $d=\sum_{q=1}^{Q} d_{q}$ is expressed as

$$
\boldsymbol{d}_{k}[n]=\left[\boldsymbol{d}_{1, k}^{\mathrm{T}}[n] \boldsymbol{d}_{2, k}^{\mathrm{T}}[n] \cdots \boldsymbol{d}_{Q, k}^{\mathrm{T}}[n]\right]^{\mathrm{T}},
$$

where $\boldsymbol{d}_{q, k}[n] \in \mathbb{R}^{d_{q}}$ denotes the desired signal for the $q$ th user on the $k$ th subcarrier and at the $n$th time instant when $(k+n)$ is even ${ }^{\mathrm{c}}$, and $d_{q}$ denotes the number of spatial streams sent to the $q$ th user. The terms $c_{i \ell} \boldsymbol{d}_{\ell}[i]$ in (1) contribute to the intrinsic interference and are pure imaginary if the prototype pulse satisfies the perfect reconstruction property $[7,12]$, where $\ell=k-1, k, k+1, i=n-3, \ldots, n+$ 3 , and $(\ell, i) \neq(k, n)$. The coefficients $c_{i \ell}(c f$. Table 1$)$ represent the system impulse response determined by the synthesis and analysis filters. The PHYDYAS prototype filter [25] is used, and the overlapping factor is chosen to be $K=4$. For more details about FBMC/OQAM systems, the reader is referred to [7]. Moreover, $\boldsymbol{n}_{k}[n] \in$ $\mathbb{C}^{M_{\mathrm{R}}^{\text {(tot) }}}$ denotes the combined additive white Gaussian noise vector with variance $\sigma_{n}^{2}$, and the noise is assumed to be spatially uncorrelated. For the $q$ th user, the noise autocorrelation matrix is written as $\sigma_{n}^{2} \boldsymbol{I}_{M_{\mathrm{R}_{q}}}$.

Furthermore, $\boldsymbol{F}_{k}[n] \in \mathbb{C}^{M_{\mathrm{T}}^{(\mathrm{BS})} \times d}$ contains the precoding matrices for all users

$$
\boldsymbol{F}_{k}[n]=\left[\boldsymbol{F}_{1, k}[n] \boldsymbol{G}_{1, k}[n] \boldsymbol{F}_{2, k}[n] \boldsymbol{G}_{2, k}[n] \cdots \boldsymbol{F}_{Q, k}[n] \boldsymbol{G}_{Q, k}[n]\right],
$$

where $\boldsymbol{F}_{q, k}[n] \in \mathbb{C}^{M_{\mathrm{T}}^{(\mathrm{BS})} \times M_{\mathrm{T}_{q}}^{(\mathrm{eq})}, q=1,2, \ldots, Q \text { are calcu- }}$ lated to mitigate the multi-user interference by employing, e.g., BD [14] such that a multi-user MIMO downlink system is decoupled into parallel equivalent single-user

\begin{tabular}{|c|c|c|c|c|c|c|c|}
\hline & $n-3$ & $n-2$ & $n-1$ & $n$ & $n+1$ & $n+2$ & $n+3$ \\
\hline$k-1$ & $0.043 \mathrm{~J}$ & -0.125 & $-0.206 J$ & 0.239 & $0.206 J$ & -0.125 & -0.043 \\
\hline k & -0.067 & 0 & 0.564 & 1 & 0.564 & 0 & -0.067 \\
\hline$k+1$ & $-0.043 J$ & -0.125 & $0.206 J$ & 0.239 & $-0.206 J$ & -0.125 & $0.043 J$ \\
\hline
\end{tabular}
transmissions. Here, $M_{\mathrm{T}_{q}}^{(\mathrm{eq})}$ symbolizes the resulting equivalent number of transmit antennas for the $q$ th user. It is

The PHYDYAS prototype filter [25] is used with the overlapping factor $K=4$. determined by the precoding scheme employed to suppress the multi-user interference, which will be explained in detail in Section 3. In addition, $\boldsymbol{G}_{q, k}[n] \in \mathbb{C}^{M_{\mathrm{T}_{q}}^{(\mathrm{eq})} \times d_{q}}$, $q=1,2, \ldots, Q$ are the transmit beamforming matrices for the equivalent single-user systems. Note that throughout this work, equal power allocation on the spatial streams and subcarriers is assumed.

\subsection{Straightforward extension of the transmission strategy as in case of CP-OFDM}

In some publications on MIMO FBMC/OQAM systems, such as [8] and [9], it is assumed that the channel stays constant across adjacent subcarriers and during consecutive symbol periods, i.e.,

$$
\begin{gathered}
\boldsymbol{H}_{k}[n]=\boldsymbol{H}_{\ell}[i], \\
\ell=k-1, k, k+1, \quad i=n-3, \ldots, n+3 .
\end{gathered}
$$

Since the precoding is performed on a per-subcarrier basis, i.e., the calculation of the precoding matrices for a certain subcarrier is solely determined by the channel on the same subcarrier, the precoding matrices are also the same on adjacent subcarriers. Therefore, the combined received signal on the $k$ th subcarrier and at the $n$th time instant can be expressed as

$$
\boldsymbol{y}_{k}[n]=\boldsymbol{H}_{k}[n] \boldsymbol{F}_{k}[n] \tilde{\boldsymbol{d}}_{k}[n]+\boldsymbol{n}_{k}[n],
$$

where $\tilde{\boldsymbol{d}}_{k}[n]$ contains the real-valued desired signal and the pure imaginary intrinsic interference

$$
\tilde{\boldsymbol{d}}_{k}[n]=\boldsymbol{d}_{k}[n]+\sum_{i=n-3}^{n+3} \sum_{\ell=k-1}^{k+1} c_{i \ell} \boldsymbol{d}_{\ell}[i], \quad(\ell, i) \neq(k, n) .
$$

Considering $\tilde{\boldsymbol{d}}_{k}[n]$ as an equivalent transmitted signal, (5) resembles the data model of a CP-OFDM-based multiuser MIMO downlink system. Consequently, transmission strategies that have been developed for multi-user MIMO CP-OFDM downlink systems can be straightforwardly extended to their FBMC/OQAM-based counterparts where only one additional step is required, i.e., taking the real part of the resulting signal after the multiplication by the decoding matrix

$$
\hat{\boldsymbol{d}}_{k}[n]=\operatorname{Re}\left\{\boldsymbol{D}_{k}^{\mathrm{H}}[n] \boldsymbol{y}_{k}[n]\right\},
$$

where $\boldsymbol{D}_{k}[n] \in \mathbb{C}^{M_{\mathrm{R}}^{(\mathrm{tot})} \times d}$ is the combined block-diagonal decoding matrix on the $k$ th subcarrier and at the $n$th time instant that contains the decoding matrices $\boldsymbol{D}_{q, k}[n] \in$ $\mathbb{C}^{M_{\mathrm{R}_{q}} \times d_{q}}, q=1,2, \ldots, Q$, for the $Q$ users, respectively. It is worth mentioning that there is no cooperation among the users, and the decoding matrix for each user is computed separately.

The concept of this transmission scheme is simple, does not induce much additional processing compared to 
CP-OFDM-based systems, and directly applies the stateof-the-art transmit as well as receive processing techniques developed for the CP-OFDM-based multi-user MIMO downlink. Nevertheless, it relies on the impractical assumption that the channel is flat fading and time invariant. In case of frequency selective channels, this transmission strategy fails to completely eliminate the intrinsic interference inherent in FBMC/OQAM systems and thus suffers from a performance degradation as also shown in the simulations section.

\subsection{Block diagonalization-based approach}

In [13] a BD-based precoding algorithm has been proposed for FBMC/OQAM-based multi-user MIMO downlink systems, where $M_{\mathrm{T}}^{(\mathrm{BS})} \geq M_{\mathrm{R}}^{(\mathrm{tot})}$. First, the $\mathrm{BD}$ algorithm [14] is used to calculate the first part of the precoding matrix $\boldsymbol{F}_{q, k}[n], q=1,2, \ldots, Q$, for the $Q$ users to mitigate the multi-user interference. By rendering $\boldsymbol{F}_{q, k}[n]$ for the $q$ th user to lie in the null space of all the other users' combined channel matrix, it is ensured that

$$
\boldsymbol{H}_{g, k}[n] \cdot \boldsymbol{F}_{q, k}[n]=\mathbf{0} \quad \in \mathbb{C}^{M_{\mathrm{R}_{q}} \times M_{\mathrm{T}_{q}}^{(\mathrm{eq})}}, \quad g \neq q .
$$

Consequently, the received signal of the $q$ th user is expressed as

$$
\begin{aligned}
\boldsymbol{y}_{q, k}[n]= & \boldsymbol{H}_{q, k}[n] \boldsymbol{F}_{q, k}[n] \boldsymbol{G}_{q, k} \boldsymbol{d}_{q, k}[n] \\
& +\sum_{i=n-3}^{n+3} \sum_{\ell=k-1}^{k+1} \boldsymbol{H}_{q, \ell}[i] \boldsymbol{F}_{q, \ell}[i] \boldsymbol{G}_{q, \ell}[i] c_{i \ell} \boldsymbol{d}_{q, \ell}[i] \\
& +\boldsymbol{n}_{q, k}[n], \quad(\ell, i) \neq(k, n),
\end{aligned}
$$

where $\boldsymbol{H}_{q, k}[n] \boldsymbol{F}_{q, k}[n] \in \mathbb{C}^{M_{\mathrm{R}_{q}} \times M_{\mathrm{T}_{q}}^{(\mathrm{eq})}}, q=1,2, \ldots, Q$, can be treated as the equivalent channels for parallel singleuser transmissions. Let us expand the real part of the received signal of the $q$ th user

$$
\begin{aligned}
\operatorname{Re}\left\{\boldsymbol{y}_{q, k}[n]\right\}= & \operatorname{Re}\left\{\boldsymbol{H}_{q, k}[n] \boldsymbol{F}_{q, k}[n] \boldsymbol{G}_{q, k}\right\} \boldsymbol{d}_{q, k}[n] \\
+ & (-1) \cdot \sum_{i=n-3}^{n+3} \sum_{\ell=k-1}^{k+1} \operatorname{Im}\left\{\boldsymbol{H}_{q, \ell}[i] \boldsymbol{F}_{q, \ell}[i] \boldsymbol{G}_{q, \ell}[i]\right\} \\
& \times \operatorname{Re}\left\{c_{i \ell} \boldsymbol{d}_{q, \ell}[i]\right\} \\
+ & \operatorname{Re}\left\{\boldsymbol{n}_{q, k}[n]\right\}, \quad(\ell, i) \neq(k, n) .
\end{aligned}
$$

To cancel the intrinsic interference, the second term on the right-hand side of (10) should be rendered as zero. This can be achieved by computing the second part of the precoding matrix such that [13]

$$
\operatorname{Im}\left\{\boldsymbol{H}_{q} \boldsymbol{F}_{q} \boldsymbol{G}_{q}\right\}=\mathbf{0} \quad \in \mathbb{C}^{M_{\mathrm{R}_{q}} \times d_{q}},
$$

where $\boldsymbol{F}_{q} \boldsymbol{G}_{q}$ represents the precoding matrix for the $q$ th user on a certain subcarrier and at a certain time instant, and $\boldsymbol{H}_{q}$ denotes the channel matrix for the $q$ th user on the same subcarrier and at the same time instant. From now on, the time and frequency indices are ignored as the precoding is performed on a per-subcarrier basis. It should be noted that the optimization of the power allocation is originally incorporated in the BD-based technique [13]. Nevertheless, this part of the algorithm is not reviewed in detail here, since optimizing the power allocation is beyond the scope of this paper and equal power allocation is assumed. In the simulations section, when using the BD-based technique as a benchmark scheme, equal power allocation is also adopted to ensure a fair comparison.

This approach outperforms the straightforward extension of the CP-OFDM case in the sense that it is able to tolerate a certain level of the frequency selectivity of the channel. However, it suffers from the dimensionality constraint that the number of transmit antennas at the base station has to be larger than or equal to the total number of receive antennas of the users, i.e., $M_{\mathrm{T}}^{(\mathrm{BS})} \geq M_{\mathrm{R}}^{(\mathrm{tot})}$. For the case where $M_{\mathrm{T}}^{(\mathrm{BS})}=M_{\mathrm{R}}^{(\mathrm{tot})}$, this scheme is not able to provide a satisfactory performance as shown later in the simulations section.

\section{Coordinated beamforming for the single-cell multi-user MIMO downlink}

Based on the philosophy on the precoding design for multi-user MIMO downlink settings first proposed in [26], the precoding matrix for each user is decomposed into two parts as already shown in (4). For the $q$ th user, the first part $\boldsymbol{F}_{q}$ eliminates the multi-user interference ${ }^{\mathrm{d}}$. The second part $\boldsymbol{G}_{q}$ plays the role of the transmit beamforming matrix for each equivalent single-user transmission after the multi-user interference cancelation. Due to the fact that the intrinsic interference resides in FBMC/OQAMbased systems, we propose to further decouple $\boldsymbol{G}_{q}$ into two parts, i.e.,

$$
\boldsymbol{G}_{q}=\boldsymbol{G}_{q, 1} \boldsymbol{G}_{q, 2} \in \mathbb{C}^{M_{\mathrm{T}_{q}}^{(\mathrm{eq})} \times d_{q}}
$$

where $\boldsymbol{G}_{q, 1} \in \mathbb{C}^{M_{\mathrm{T}_{q}}^{(\mathrm{eq})} \times M_{x_{q}}}$ is computed to suppress the intrinsic interference, and $\boldsymbol{G}_{q, 2} \in \mathbb{R}^{M_{x_{q}} \times d_{q}}$ is used for the spatial mapping.

\subsection{The IIM-CBF 1 algorithm}

We first propose a coordinated beamforming-based transmission scheme IIM-CBF 1 for symmetric multi-user MIMO downlink settings where $M_{\mathrm{T}}^{(\mathrm{BS})}=M_{\mathrm{R}}^{(\mathrm{tot})}$. BD [14] is employed to calculate the first part of the precoding matrices $\boldsymbol{F}_{q} \in \mathbb{C}^{M_{\mathrm{T}}^{(\mathrm{BS})} \times M_{\mathrm{T}_{q}}^{(\mathrm{eq})}, q=1,2, \ldots, Q \text {. For the } q \text { th }}$ user, define a matrix $\widetilde{\boldsymbol{H}}_{\mathrm{e}} \in \mathbb{C}^{\left(M_{\mathrm{R}}^{(\mathrm{tot})}-M_{\mathrm{R} q}\right) \times M_{\mathrm{T}}^{(\mathrm{BS})}}$ as

$$
\widetilde{\boldsymbol{H}}_{\mathrm{e}}=\left[\begin{array}{lllll}
\boldsymbol{H}_{1}^{\mathrm{T}} & \ldots & \boldsymbol{H}_{q-1}^{\mathrm{T}} & \boldsymbol{H}_{q+1}^{\mathrm{T}} \ldots \boldsymbol{H}_{\mathrm{Q}}^{\mathrm{T}}
\end{array}\right]^{\mathrm{T}},
$$

which contains the channel matrices of all the other users. The precoding matrix $\boldsymbol{F}_{q}$ for the $q$ th user is obtained as 
$\boldsymbol{F}_{q}=\widetilde{\boldsymbol{V}}_{\mathrm{e}_{(q, 0)}} \in \mathbb{C}^{M_{\mathrm{T}}^{(\mathrm{BS})} \times M_{\left.\mathrm{T}_{q}\right)}^{(\mathrm{eq})}}$, where $\widetilde{\boldsymbol{V}}_{\mathrm{e}_{(q, 0)}}$ contains the last $M_{\mathrm{T}_{q}}^{(\mathrm{eq})}$ right singular vectors that form an orthonormal basis for the null space of $\widetilde{\boldsymbol{H}}_{\mathrm{e}}$ [14]. The resulting equivalent number of transmit antennas $M_{\mathrm{T}_{q}}^{(\mathrm{eq})}=M_{\mathrm{T}}^{(\mathrm{BS})}-$ $\sum_{g=1, g \neq q}^{Q} M_{\mathrm{R}_{g}}$ is equal to $M_{\mathrm{R}_{q}}$. The reader is referred [14] for more details of the $\mathrm{BD}$ algorithm.

For the $q$ th user, we propose to jointly and iteratively update the second part of its precoding matrix $\boldsymbol{G}_{q}$ and its decoding matrix. An equivalent channel matrix $\boldsymbol{H}_{\mathrm{e}_{q}}$ is defined as

$$
\boldsymbol{H}_{\mathrm{e}_{q}}=\boldsymbol{D}_{q}^{\mathrm{T}} \boldsymbol{H}_{q} \boldsymbol{F}_{q} \in \mathbb{C}^{d_{q} \times M_{\mathrm{T}_{q}}^{(\mathrm{eq})}},
$$

where $\boldsymbol{D}_{q} \in \mathbb{R}^{M_{\mathrm{R}_{q}} \times d_{q}}$ is the real-valued decoding matrix.

The proposed coordinated beamforming algorithm is summarized as follows:

- Step 1: Initialize the decoding matrix $\boldsymbol{D}_{q}^{(0)} \in \mathbb{R}^{M_{\mathrm{R}_{q}} \times d_{q}}$, set the iteration index $p$ to zero, and set a threshold $\epsilon$ for the stopping criterion. The decoding matrix is generated randomly if the current subcarrier is the first one; otherwise, set the decoding matrix as the one calculated for the previous subcarrier [24].

- Step 2: Set $p \leftarrow p+1$ and calculate the equivalent channel matrix $\boldsymbol{H}_{\mathrm{e}_{q}}^{(p)}$ in the $p$ th iteration as

$$
\boldsymbol{H}_{\mathrm{e}_{q}}^{(p)}=\boldsymbol{D}_{q}^{(p-1)^{\mathrm{T}}} \boldsymbol{H}_{q} \boldsymbol{F}_{q} \in \mathbb{C}^{d_{q} \times M_{\mathrm{T}_{q}}^{(\mathrm{eq})}} .
$$

Define a matrix

$$
\check{\boldsymbol{H}}_{\mathrm{e}_{q}}^{(p)}=\left[\operatorname{Im}\left\{\boldsymbol{H}_{\mathrm{e}_{q}}^{(p)}\right\} \operatorname{Re}\left\{\boldsymbol{H}_{\mathrm{e}_{q}}^{(p)}\right\}\right] \in \mathbb{R}^{d_{q} \times 2 M_{\mathrm{T}_{q}}^{(\mathrm{eq})}} .
$$

- Step 3: Calculate the precoding matrix $\boldsymbol{G}_{q}^{(p)}=\boldsymbol{G}_{q, 1}^{(p)} \boldsymbol{G}_{q, 2}^{(p)}$ for the $p$ th iteration. First, we perform the singular value decomposition (SVD) of $\check{\boldsymbol{H}}_{\mathrm{e}_{q}}^{(p)}$ as

$$
\check{\boldsymbol{H}}_{\mathrm{e}_{q}}^{(p)}=\boldsymbol{U}_{q, 1}^{(p)} \boldsymbol{\Sigma}_{q, 1}^{(p)} \boldsymbol{V}_{q, 1}^{(p)^{\mathrm{T}}}
$$

Denoting the rank of $\check{\boldsymbol{H}}_{\mathrm{e}_{q}}^{(p)}$ as $r_{q}^{(p)}$, we define $V_{q, 1,0}^{(p)} \in \mathbb{R}^{2 M_{\mathrm{T}_{q}}^{(\mathrm{eq})} \times M_{x_{q}}}$ as containing the last $M_{x_{q}}=2 M_{\mathrm{T}_{q}}^{(\mathrm{eq})}-r_{q}^{(p)}$ right singular vectors that form an orthonormal basis for the null space of $\check{\boldsymbol{H}}_{\mathrm{e}_{q}}^{(p)}$. Hence, $\boldsymbol{G}_{q, 1}^{(p)}$ for the $p$ th iteration can be obtained via

$$
\boldsymbol{V}_{1,0}^{(p)}=\left[\begin{array}{c}
\operatorname{Re}\left\{\boldsymbol{G}_{q, 1}^{(p)}\right\} \\
\operatorname{Im}\left\{\boldsymbol{G}_{q, 1}^{(p)}\right\}
\end{array}\right] \in \mathbb{R}^{2 M_{\mathrm{T}_{q}}^{(\mathrm{eq})} \times M_{x_{q}}} .
$$

To further calculate $\boldsymbol{G}_{q, 2}^{(p)}$, the following equivalent channel matrix after the cancelation of the intrinsic interference for the $p$ th iteration is defined as

$$
\widetilde{\boldsymbol{H}}_{\mathrm{e}_{q}}^{(p)}=\operatorname{Re}\left\{\boldsymbol{H}_{\mathrm{e}_{q}}^{(p)} \boldsymbol{G}_{q, 1}^{(p)}\right\} \in \mathbb{R}^{d_{q} \times M_{x_{q}}} .
$$

Further calculate the SVD of $\widetilde{\boldsymbol{H}}_{\mathrm{e}_{q}}^{(p)}$ and define $V_{q, 2,1}^{(p)} \in \mathbb{R}^{M_{x_{q}} \times d_{q}}$ as containing the first $d_{q}$ right singular vectors. Thereby, $\boldsymbol{G}_{q, 2}^{(p)}$ is obtained as $\boldsymbol{G}_{q, 2}^{(p)}=\boldsymbol{V}_{q, 2,1}^{(p)}$.

- Step 4: Update the decoding matrix based on the equivalent channel matrix after the cancelation of the intrinsic interference where only the processing at the transmitter is considered

$$
\widetilde{\boldsymbol{H}}_{\operatorname{etx}_{q}}^{(p)}=\operatorname{Re}\left\{\boldsymbol{H}_{q} \boldsymbol{F}_{q} \boldsymbol{G}_{q}^{(p)}\right\} \in \mathbb{R}^{M_{\mathrm{R}_{q}} \times d_{q}} .
$$

When the MMSE receiver ${ }^{\mathrm{e}}$ is used, the decoding matrix has the following form

$$
\boldsymbol{D}_{q}^{(p)}=\widetilde{\boldsymbol{H}}_{\operatorname{etx}_{q}}^{(p)}\left(\widetilde{\boldsymbol{H}}_{\operatorname{etx}_{q}}^{(p)^{\mathrm{T}}} \tilde{\boldsymbol{H}}_{\operatorname{etx}_{q}}^{(p)}+\sigma_{n}^{2} \boldsymbol{I}_{d_{q}}\right)^{-1} .
$$

- Step 5: Calculate the term $\Delta\left(\boldsymbol{G}_{q}\right)$ defined as

$$
\Delta\left(\boldsymbol{G}_{q}\right)=\left\|\boldsymbol{G}_{q}^{(p)}-\boldsymbol{G}_{q}^{(p-1)}\right\|_{\mathrm{F}}^{2},
$$

which measures the change of the precoding matrix $\boldsymbol{G}_{q}$. If $\Delta\left(\boldsymbol{G}_{q}\right)<\epsilon$, the convergence is achieved, and the iterative procedure terminates. Otherwise go back to Step 2.

It is important to note that in the special case where $M_{\mathrm{T}_{q}}^{(\mathrm{eq})}=M_{\mathrm{R}_{q}}=d_{q}+1$, we propose to compute the decoding matrix in the initialization step as follows. Consequently, it is observed that the coordinated beamforming technique only needs two iterations to converge. First, calculate the SVD of $\check{\boldsymbol{H}}_{q} \in \mathbb{R}^{M_{\mathrm{R}_{q}} \times 2 M_{\mathrm{T}_{q}}^{(\text {(eq) }}}$

$$
\check{\boldsymbol{H}}_{q}=\left[\operatorname{Im}\left\{\boldsymbol{H}_{q} \boldsymbol{F}_{q}\right\} \operatorname{Re}\left\{\boldsymbol{H}_{q} \boldsymbol{F}_{q}\right\}\right],
$$

and let $V_{q, 1,0}^{(0)}$ contain the last $\left(2 M_{\mathrm{T}_{q}}^{(\mathrm{eq})}-M_{\mathrm{R}_{q}}\right)$ right singular vectors. Defining $\boldsymbol{G}_{q, 1}^{(0)}$ via

$$
\boldsymbol{V}_{q, 1,0}^{(0)}=\left[\begin{array}{c}
\operatorname{Re}\left\{\boldsymbol{G}_{q, 1}^{(0)}\right\} \\
\operatorname{Im}\left\{\boldsymbol{G}_{q, 1}^{(0)}\right\}
\end{array}\right],
$$

we compute $\boldsymbol{U}_{q, 2,1}^{(0)} \in \mathbb{R}^{M_{\mathrm{R}_{q}} \times d_{q}}$ from the SVD of $\operatorname{Re}\left\{\boldsymbol{H}_{q} \boldsymbol{F}_{q} \boldsymbol{G}_{q, 1}^{(0)}\right\}$ such that it contains the first $d_{q}$ left singular vectors. Then the decoding matrix for the initialization step is chosen as

$$
\boldsymbol{D}_{q}^{(0)}=\boldsymbol{U}_{q, 2,1}^{(0)} \text {. }
$$




\subsection{The IIM-CBF 2 algorithm}

In multi-user MIMO FBMC/OQAM downlink systems where the total number of receive antennas of the users exceeds the number of transmit antennas at the base station, the BD algorithm [14] or the BD-based technique [13] cannot be employed to achieve the multi-user interference or the intrinsic interference suppression.

Therefore, we propose another iterative procedure to jointly compute the precoding matrix and the decoding matrix. Let us first define an equivalent combined channel matrix after the decoding at the user terminals as

$$
\boldsymbol{H}_{\mathrm{e}}=\left[\begin{array}{c}
\boldsymbol{D}_{1}^{\mathrm{T}} \boldsymbol{H}_{1} \\
\boldsymbol{D}_{2}^{\mathrm{T}} \boldsymbol{H}_{2} \\
\vdots \\
\boldsymbol{D}_{\mathrm{Q}}^{\mathrm{T}} \boldsymbol{H}_{\mathrm{Q}}
\end{array}\right] \in \mathbb{C}^{d \times M_{\mathrm{T}}^{(\mathrm{BS})} .}
$$

Unlike the coordinated beamforming schemes in [19] or [24], the decoding matrices $\boldsymbol{D}_{q} \in \mathbb{R}^{M_{\mathrm{R}_{q}} \times d_{q}}, q=1,2, \ldots, Q$ are forced to be real-valued. Although the BD-based concept cannot be employed on the physical channel due to the dimensionality constraint, it can be used on this equivalent channel.

The proposed IIM-CBF 2 algorithm for the FBMC/OQAM-based multi-user MIMO downlink system is described in detail as follows:

- Step 1: Initialize the decoding matrices $\boldsymbol{D}_{q}^{(0)} \in \mathbb{R}^{M_{\mathrm{R}_{q}} \times d_{q}}(q=1, \ldots, Q)$, set the iteration index $p$ to zero, and set a threshold $\epsilon$ for the stopping criterion. If the current subcarrier is the first one, the decoding matrices are generated randomly; otherwise, set the decoding matrices as those calculated for the previous subcarrier [24].

- Step 2: Set $p \leftarrow p+1$ and calculate the equivalent channel matrix $\boldsymbol{H}_{\mathrm{e}}^{(p)}$ in the $p$ th iteration as

$$
\boldsymbol{H}_{\mathrm{e}}^{(p)}=\left[\begin{array}{llll}
\boldsymbol{H}_{\mathrm{e}_{1}}^{(p)^{\mathrm{T}}} & \boldsymbol{H}_{\mathrm{e}_{2}}^{(p)^{\mathrm{T}}} & \cdots & \boldsymbol{H}_{\mathrm{e}_{\mathrm{Q}}}^{(p)^{\mathrm{T}}}
\end{array}\right]^{\mathrm{T}},
$$

where $\boldsymbol{H}_{\mathrm{e}_{q}}^{(p)}=\boldsymbol{D}_{q}^{(p-1)^{\mathrm{T}}} \boldsymbol{H}_{q}$ is the equivalent channel matrix for the $q$ th user in the $p$ th iteration.

- Step 3: Calculate the precoding matrices $\boldsymbol{F}_{q}^{(p)}$ $(q=1, \ldots, Q)$ in the $p$ th iteration to cancel the multiuser interference based on the BD algorithm [14]. For the $q$ th user, define a matrix $\widetilde{\boldsymbol{H}}_{\mathrm{e}_{q}}^{(p)} \in \mathbb{C}^{\left(d-d_{q}\right) \times M_{\mathrm{T}}^{(\mathrm{BS})} \text { as }}$

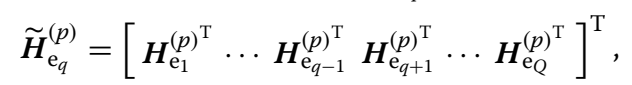

which contains the equivalent channel matrices of all the other users that are calculated in Step 2. The precoding matrix $\boldsymbol{F}_{q}^{(p)}$ for the $q$ th user in the $p$ th iteration is obtained as $\boldsymbol{F}_{q}^{(p)}=\widetilde{\boldsymbol{V}}_{\mathrm{e}(q, 0)}^{(p)} \in \mathbb{C}^{M_{\mathrm{T}}^{(\mathrm{BS})} \times M_{\mathrm{T}_{q}}^{(\mathrm{eq})}}$, where $\widetilde{\boldsymbol{V}}_{\mathrm{e}_{(q, 0)}^{(p)}}^{(p)}$ contains the last $M_{\mathrm{T}_{q}}^{(\mathrm{eq})}$ right singular vectors that form an orthonormal basis for the null space of $\widetilde{\boldsymbol{H}}_{\mathrm{e}_{q}}^{(p)}$ [14]. To this end, the multi-user MIMO downlink transmission is decoupled into parallel equivalent single-user MIMO transmissions that will be considered in the following steps.

- Step 4: Define a matrix $\check{\boldsymbol{H}}_{\mathrm{e}_{q}}^{(p)} \in \mathbb{R}^{d_{q} \times 2 M_{\mathrm{T}_{q}}^{(\mathrm{eq})}}$ for the $q$ th user based on its equivalent channel matrix $\boldsymbol{H}_{\mathrm{e}_{q}}^{(p)} \boldsymbol{F}_{q}^{(p)}$ after the cancelation of the multi-user interference

$$
\check{\boldsymbol{H}}_{\mathrm{e}_{q}}^{(p)}=\left[\operatorname{Im}\left\{\boldsymbol{H}_{\mathrm{e}_{q}}^{(p)} \boldsymbol{F}_{q}^{(p)}\right\} \operatorname{Re}\left\{\boldsymbol{H}_{\mathrm{e}_{q}}^{(p)} \boldsymbol{F}_{q}^{(p)}\right\}\right] .
$$

- Step 5: Calculate the precoding matrix $\boldsymbol{G}_{q}^{(p)}=\boldsymbol{G}_{q, 1}^{(p)} \boldsymbol{G}_{q, 2}^{(p)}$ for the $q$ th user in the $p$ th iteration. First, we perform the SVD of $\check{\boldsymbol{H}}_{\mathrm{e}_{q}}^{(p)}$ and obtain $\boldsymbol{V}_{q, 1,0}^{(p)} \in$ $\mathbb{R}^{2 M_{\mathrm{T}_{q}}^{(\mathrm{eq})} \times M_{x_{q}}}$ as containing the last $M_{x_{q}}=2 M_{\mathrm{T}_{q}}^{(\mathrm{eq})}-$ $r_{q}^{(p)}$ right singular vectors that form an orthonormal basis for the null space of $\check{\boldsymbol{H}}_{\mathrm{e}_{q}}^{(p)}$, where $r_{q}^{(p)}$ denotes the rank of $\check{\boldsymbol{H}}_{\mathrm{e}_{q}}^{(p)}$. Hence, $\boldsymbol{G}_{q, 1}^{(p)} \in \mathbb{C}^{M_{\mathrm{T}_{q}}^{(\mathrm{eq})} \times M_{x_{q}}}$ can be obtained via

$$
\boldsymbol{V}_{q, 1,0}^{(p)}=\left[\begin{array}{c}
\operatorname{Re}\left\{\boldsymbol{G}_{q, 1}^{(p)}\right\} \\
\operatorname{Im}\left\{\boldsymbol{G}_{q, 1}^{(p)}\right\}
\end{array}\right] \in \mathbb{R}^{2 M_{\mathrm{T}_{q}}^{(\mathrm{eq})} \times M_{x_{q}}}
$$

such that (11) is fulfilled to achieve the mitigation of the intrinsic interference.

Now we define the following equivalent channel matrix after canceling the intrinsic interference for the $q$ th user in the $p$ th iteration

$$
\overline{\boldsymbol{H}}_{\mathrm{e}_{q}}^{(p)}=\operatorname{Re}\left\{\boldsymbol{H}_{\mathrm{e}_{q}}^{(p)} \boldsymbol{F}_{q}^{(p)} \boldsymbol{G}_{q, 1}^{(p)}\right\} \in \mathbb{R}^{d_{q} \times M_{x_{q}}} .
$$

Further calculate the SVD of $\overline{\boldsymbol{H}}_{\mathrm{e}_{q}}^{(p)}$ and define $\boldsymbol{V}_{q, 2,1}^{(p)} \in \mathbb{R}^{M_{x_{q}} \times d_{q}}$ as containing the first $d_{q}$ right singular vectors. Then $\boldsymbol{G}_{q, 2}^{(p)}$ is obtained as $\boldsymbol{G}_{q, 2}^{(p)}=\boldsymbol{V}_{q, 2,1}^{(p)}$.

- Step 6: Update the decoding matrix for each user based on the real-valued equivalent channel matrix where the processing at the transmitter and the procedure of taking the real part of the receive signal are taken into account

$\boldsymbol{H}_{\mathrm{etx}_{q}}^{(p)}=\operatorname{Re}\left\{\boldsymbol{H}_{q} \boldsymbol{F}_{q}^{(p)} \boldsymbol{G}_{q}^{(p)}\right\} \in \mathbb{R}^{M_{\mathrm{R}_{q}} \times d_{q}}, \quad q=1, \ldots, Q$.

When the MMSE receiver is used, the update of the decoding matrix in the $p$ th iteration for the $q$ th user has the following form:

$$
\boldsymbol{D}_{q}^{(p)}=\boldsymbol{H}_{\operatorname{etx}_{q}}^{(p)}\left(\boldsymbol{H}_{\operatorname{etx}_{q}}^{(p)^{\mathrm{T}}} \boldsymbol{H}_{\mathrm{etx}_{q}}^{(p)}+\sigma_{n}^{2} \boldsymbol{I}_{d_{q}}\right)^{-1} .
$$


- Step 7: Calculate the term $\xi^{(p)}$ that measures the residual multi-user and the inter-stream interference for the $p$ th iteration defined as

$$
\xi^{(p)}=\left\|\operatorname{off}\left(\boldsymbol{D}^{(p)^{\mathrm{T}}} \operatorname{Re}\left\{\boldsymbol{H} \boldsymbol{F}^{(p)}\right\}\right)\right\|_{\mathrm{F}}^{2}
$$

where off $(\cdot)$ indicates an operation of keeping all off-diagonal elements of its input matrix while setting its diagonal elements to zero. If $\xi^{(p)}<\epsilon$, the convergence is achieved, and the iterative procedure terminates. Otherwise go back to Step 2.

Note that the stopping criterion similar as (22) that tracks the change of the precoding matrix can also be adopted $^{f}$. In addition, for both IIM-CBF schemes proposed in this section and Section 3.1, it is not required that the users are informed of the decoding matrices that are obtained at the base station while computing the precoding matrices. After the users acquire the information of the effective channel via channel estimation, the receive processing can be performed. For example, the MMSE receiver of the effective channel for each user can be employed.

\section{Coordinated beamforming for the CoMP downlink}

In this section, we focus on a CoMP downlink setting based on [19]. Note that in [19] a clustered cellular scenario is considered where each cluster contains multiple cells. Since full cooperation is assumed in each cluster, the downlink transmissions for each cluster resemble those of the single-cell multi-user MIMO downlink. Therefore, we simplify the scenario description of the CoMP downlink and only consider joint transmission of adjacent cells. It is assumed that the cell interior users only receive signals from their own base station and suffer only from the intracell interference, i.e., the multi-user interference as in the single-cell multi-user MIMO downlink scenarios. On the other hand, both the intra-cell interference and the intercell interference have an impact on the cell edge users. To assist the cell edge users to combat the interference and also deal with the greater path loss compared to the cell interior users, the base stations of the adjacent cells transmit the same signals to each cell edge user. An example of a two-cell FBMC/OQAM-based CoMP downlink scenario is illustrated in Figure 1.

Assume that Cell 1 and Cell 2 are equipped with $M_{\mathrm{T}}^{(\mathrm{BS} 1)}$ and $M_{\mathrm{T}}^{(\mathrm{BS} 2)}$ transmit antennas, respectively. The number of users served by each cell is denoted as $Q$. The received signal on the $k$ th subcarrier and at the $n$th time instant of the $q$ th user of Cell 1 as a cell interior user is expressed as

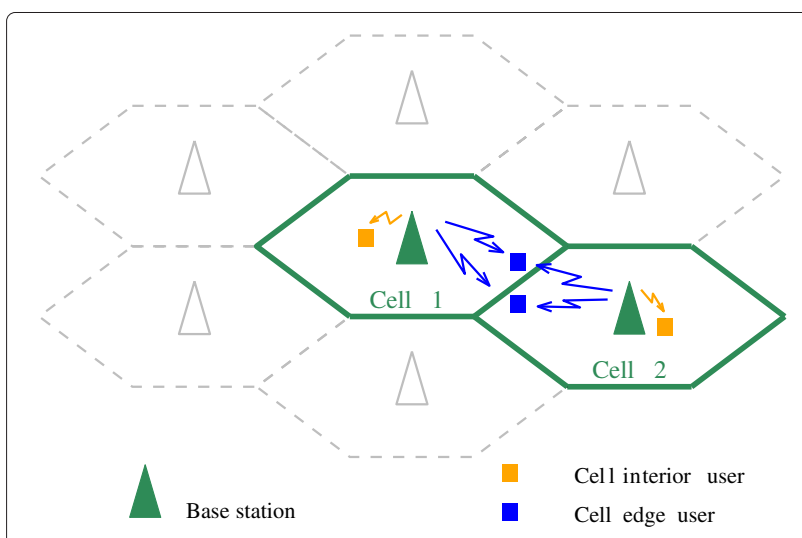

Figure 1 Illustration of a two-cell CoMP downlink scenario. An example of a two-cell CoMP downlink scenario where a cell interior user and two cell edge users are served in each cell.

$$
\begin{aligned}
\boldsymbol{y}_{q, k}[n]= & \boldsymbol{H}_{q, k,(1)}[n] \boldsymbol{F}_{k,(1)}[n] \boldsymbol{d}_{k,(1)}[n] \\
& +\sum_{i=n-3}^{n+3} \sum_{\ell=k-1}^{k+1} \boldsymbol{H}_{q, \ell,(1)}[i] \boldsymbol{F}_{\ell,(1)}[i] c_{i \ell} \boldsymbol{d}_{\ell,(1)}[i] \\
& +\boldsymbol{n}_{q, k}[n], \quad(\ell, i) \neq(k, n),
\end{aligned}
$$

where $\boldsymbol{H}_{q, k,(1)}[n] \in \mathbb{C}^{M_{\mathrm{R}_{q}} \times M_{\mathrm{T}}^{(\mathrm{BS} 1)}}$ represents the channel matrix between the base station of Cell 1 and the $q$ th user with $M_{\mathrm{R}_{q}}$ receive antennas, $\boldsymbol{F}_{k,(1)}[n]$ symbolizes the precoding matrix for Cell 1 , and $\boldsymbol{d}_{k,(1)}[n]$ contains the signals for all users served by Cell 1 . It can be seen that for a cell interior user, assuming that the inter-cell interference is negligible, the transmission from the base station in its own cell is the same as in a single-cell multi-user MIMO downlink system. For the $g$ th user that is a cell edge user and receives the same signals from both Cell 1 and Cell 2 , its received signal on the $k$ th subcarrier and at the $n$th time instant has the following form:

$$
\begin{aligned}
\boldsymbol{y}_{g, k}[n]= & \boldsymbol{H}_{g, k,(1)}[n] \boldsymbol{F}_{k,(1)}[n] \boldsymbol{d}_{k,(1)}[n] \\
& +\sum_{i=n-3}^{n+3} \sum_{\ell=k-1}^{k+1} \boldsymbol{H}_{g, \ell,(1)}[i] \boldsymbol{F}_{\ell,(1)}[i] c_{i \ell} \boldsymbol{d}_{\ell,(1)}[i] \\
& +\boldsymbol{H}_{g, k,(2)}[n] \boldsymbol{F}_{k,(2)}[n] \boldsymbol{d}_{k,(2)}[n] \\
& +\sum_{i=n-3}^{n+3} \sum_{\ell=k-1}^{k+1} \boldsymbol{H}_{g, \ell,(2)}[i] \boldsymbol{F}_{\ell,(2)}[i] c_{i \ell} \boldsymbol{d}_{\ell,(2)}[i] \\
& +\boldsymbol{n}_{g, k}[n], \quad(\ell, i) \neq(k, n) .
\end{aligned}
$$

Here the channel matrix, precoding matrix, and the data vector with respect to Cell 2 are denoted similarly as in (35) only with '(2)' in the subscripts. Note that $\boldsymbol{d}_{g, k,(1)}[n]=$ 
$\boldsymbol{d}_{g, k,(2)}[n]$, i.e., the signals from Cell 1 and Cell 2 transmitted to the $g$ th user ${ }^{g}$ are the same. To enable such FBMC/OQAM-based CoMP downlink transmissions, the mitigation of the intra-cell, inter-cell, and intrinsic interference has to be achieved. Therefore, we propose the following IIM-CBF scheme that is an extension of the approach described in Section 3.2 and is also the outcome of adapting the Extended FlexCoBF algorithm for CP-OFDM-based systems in [19] to FBMC/OQAM-based systems.

Consider $M$ cells, and the $m$ th cell serves $Q_{m}$ users simultaneously, $m=1,2, \ldots, M$. It is assumed that for the $m$ th cell, the users $1,2, \ldots, L_{m}$ are cell interior users, while the remaining $\left(Q_{m}-L_{m}\right)$ users are cell edge users. The proposed IIM-CBF 3 scheme is summarized as follows:

- Step 1: Initialize the real-valued decoding matrices $\boldsymbol{D}_{q}^{(0)}\left(q=1, \ldots, Q_{m}\right)$ for the $m$ th cell, where $m=1, \ldots, M$. Set the iteration index $p$ to zero, and set a threshold $\epsilon$ for the stopping criterion. If the current subcarrier is the first one, the decoding matrices are generated randomly; otherwise, set the decoding matrices as those calculated for the previous subcarrier [24].

- Step 2: Set $p \leftarrow p+1$ and calculate the equivalent channel matrix $\boldsymbol{H}_{\mathrm{e}_{m}}^{(p)}$ in the $p$ th iteration as

$$
\boldsymbol{H}_{\mathrm{e}_{m}}^{(p)}=\left[\begin{array}{llll}
\boldsymbol{H}_{\mathrm{e}_{(1, m)}}^{(p)^{\mathrm{T}}} & \boldsymbol{H}_{\mathrm{e}_{(2, m)}}^{(p)^{\mathrm{T}}} \cdots \boldsymbol{H}_{\mathrm{e}_{\left(Q_{m}, m\right)}^{(p)^{\mathrm{T}}}}^{()^{2}}
\end{array}\right]^{\mathrm{T}}
$$

where $\boldsymbol{H}_{\mathrm{e}_{(q, m)}^{(p)}}^{(p)}=\boldsymbol{D}_{q}^{(p-1)^{\mathrm{T}}} \boldsymbol{H}_{q, m}$ is the equivalent channel matrix for the $q$ th user in the $p$ th iteration.

- Step 3: Calculate the precoding matrices $\boldsymbol{F}_{q, m}^{(p)} \boldsymbol{G}_{q, m}^{(p)}$ $\left(q=1, \ldots, Q_{m}\right)$ following Step 3 to Step 5 of Section 3.2 to achieve the suppression of the multi-user interference and the intrinsic interference.

- Step 4: Update the decoding matrix for each user based on the real-valued equivalent channel matrix where the processing at the transmitter and the procedure of taking the real part of the receive signal are taken into account.

1. For the $q$ th user that is a cell interior user of the $m$ th cell, its equivalent channel matrix $\boldsymbol{H}_{\operatorname{et}_{(q, m)}^{(p)}}^{(i)}$ calculated as

$$
\boldsymbol{H}_{\mathrm{etx}_{(q, m)}^{(p)}}^{(p)}=\operatorname{Re}\left\{\boldsymbol{H}_{q, m} \boldsymbol{F}_{q, m}^{(p)} \boldsymbol{G}_{q, m}^{(p)}\right\} .
$$

2. For the $q$ th user that is a cell edge user, define a set $\mathcal{S}_{q, m}$ that contains the indices of the cells that simultaneously transmit the same signals to the $q$ th user. Then its equivalent channel matrix is expressed as

$$
\boldsymbol{H}_{\mathrm{etx}(q, m)}^{(p)}=\sum_{r \in \mathcal{S}_{q, m}} \operatorname{Re}\left\{\boldsymbol{H}_{q_{r}, r} \boldsymbol{F}_{q_{r}, r}^{(p)} \boldsymbol{G}_{q_{r}, r}^{(p)}\right\}
$$

where $q_{r}$ represents the index of the $q$ th user of the $m$ th cell in the $r$ th cell and $q_{m}=q$ following this definition. It is required that the base station of the $m$ th cell is informed by the $r$ th cell, $r \in \mathcal{S}_{q, m}$ and $r \neq m$, of the corresponding real-valued equivalent channel matrices after the precoding and the operation of taking the real part of the received signal. In a summary, the cooperation of the adjacent cells involves the knowledge of the signals for cell edge users. It also requires the exchange of these real-valued equivalent channel matrices that are used to compute the decoding matrix for each cell edge user, which can be achieved by adopting the two exchange mechanisms proposed in [19].

Afterwards, when a single data stream is transmitted to each user, the decoding matrix for the $q$ th user in the $p$ th iteration can be obtained by employing the maximal ratio combining (MRC) receiver or the MMSE receiver of the its equivalent channel matrix

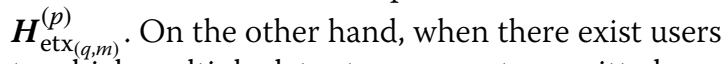
to which multiple data streams are transmitted, we propose to use the $\mathrm{ZF}$ receiver ${ }^{\mathrm{h}}$

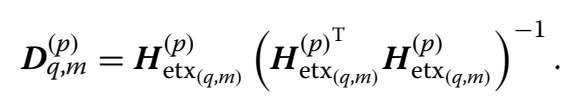

- Step 5: Calculate the term $\xi_{m}^{(p)}$ for the $m$ th cell that measures the residual multi-user and the inter-stream interference for the $p$ th iteration. When a single data stream is transmitted to each user, $\xi_{m}^{(p)}$ is defined as

$$
\xi_{m}^{(p)}=\left\|\operatorname{off}\left(\boldsymbol{D}_{m}^{(p)^{\mathrm{T}}} \cdot \operatorname{Re}\left\{\boldsymbol{H}_{m} \boldsymbol{F}_{m}^{(p)}\right\}\right)\right\|_{\mathrm{F}}^{2}
$$

where $\operatorname{off}(\cdot)$ indicates an operation of keeping all offdiagonal elements of its input matrix while setting its diagonal elements to zero. Moreover, $\boldsymbol{D}_{m}^{(p)}, \boldsymbol{H}_{m}$, and $\boldsymbol{F}_{m}^{(p)}$ denote the block diagonal combined decoding matrix in the $p$ th iteration, the combined channel matrix, and the precoding matrix in the $p$ th iteration for the $m$ th cell, respectively.

When multiple data streams are transmitted to at least one of the cell edge users, we propose to express the term $\xi_{m}^{(p)}$ as 


$$
\begin{aligned}
\xi_{m}^{(p)}=\sum_{q=1}^{L_{m}} & \left(\left\|\operatorname{off}\left(\boldsymbol{D}_{q}^{(p)^{\mathrm{T}}} \cdot \operatorname{Re}\left\{\boldsymbol{H}_{q, m} \boldsymbol{F}_{q, m}^{(p)} \boldsymbol{G}_{q, m}^{(p)}\right\}\right)\right\|_{\mathrm{F}}^{2}\right. \\
& \left.+\sum_{g=1, g \neq q}^{Q_{m}}\left\|\boldsymbol{D}_{q}^{(p)^{\mathrm{T}}} \cdot \operatorname{Re}\left\{\boldsymbol{H}_{q, m} \boldsymbol{F}_{g, m}^{(p)} \boldsymbol{G}_{g, m}^{(p)}\right\}\right\|_{\mathrm{F}}^{2}\right) \\
& +\sum_{q=L_{m}+1}^{Q_{m}}\left(\left\|\operatorname{off}\left(\boldsymbol{D}_{q}^{(p)^{\mathrm{T}}} \cdot \sum_{r \in \mathcal{S}_{q, m}} \operatorname{Re}\left\{\boldsymbol{H}_{q_{r}, r} \boldsymbol{F}_{q, r}^{(p)} \boldsymbol{G}_{q_{r}, r}^{(p)}\right\}\right)\right\|_{\mathrm{F}}^{2}\right. \\
& \left.+\sum_{g=1, g \neq q}^{Q_{m}}\left\|\boldsymbol{D}_{q}^{(p)^{\mathrm{T}}} \cdot \operatorname{Re}\left\{\boldsymbol{H}_{q, m} \boldsymbol{F}_{g, m}^{(p)} \boldsymbol{G}_{g, m}^{(p)}\right\}\right\|_{\mathrm{F}}^{2}\right) .
\end{aligned}
$$

On the right-hand side of (42), the first term corresponds to the residual inter-stream interference of each cell interior user, while the second term represents the residual multi-user interference that it still suffers from. For each cell edge user, the third term on the right-hand side of (42) measures its residual inter-stream interference. Here we take into account the fact that coordinated adjacent cells transmit the same signals to the cell edge user simultaneously. Recall that $\mathcal{S}_{q, m}$ contains the indices of the cells that serve the $q$ th user of the $m$ th cell. Moreover, the fourth term corresponds to the residual multiuser interference that affects each cell edge user. If $\xi_{m}^{(p)}<$ $\epsilon$, the convergence is achieved, and the iterative procedure terminates. Otherwise go back to Step 2.

As mentioned before, this coordinated beamforming scheme is designed based on the CoMP technique in [19]. Nevertheless, due to the fact that the intrinsic interference is inherent in FBMC/OQAM systems, we have proposed to incorporate the additional processing to suppress the intrinsic interference. Moreover, different choices of the stopping criterion are recommended for singlestream transmissions and multiple-stream transmissions, respectively.

Finally, we summarize the proposed IIM-CBF schemes in Table 2. These three algorithms are all based on the concept of jointly and iteratively computing the precoding matrix and decoding matrix. In addition, they mitigate the intra-cell and inter-cell interference as well as the intrinsic interference in a ZF fashion. Nevertheless, the three

Table 2 Acronyms of the proposed IIM-CBF schemes and the corresponding scenarios

\begin{tabular}{lll}
\hline Acronyms & Configurations & Interference type \\
\hline IIM-CBF 1 & $\begin{array}{l}\text { Multi-user MIMO downlink, } \\
M_{T}^{(B S)}=M_{R}^{(t o t)}\end{array}$ & Intrinsic, multi-user \\
IIM-CBF 2 & $\begin{array}{ll}\text { Multi-user MIMO downlink, } & \text { Intrinsic, multi-user } \\
& M_{T}^{(B S)}<M_{R}^{(t o t)}\end{array}$ \\
IIM-CBF 3 & CoMP downlink & Intrinsic, intra-/inter-cell
\end{tabular}

schemes are designed for different configurations and deal with the different types of interference. IIM-CBF 1 is developed for symmetric single-cell FBMC/OQAM-based multi-user MIMO downlink settings. In this scenario, BD can still be employed to cancel the multi-user interference. Therefore, in IIM-CBF 1 only the precoding matrix for each equivalent single user transmission (i.e., $\boldsymbol{G}_{q}$ for the $q$ th user) is computed via the iterative procedure to suppress the intrinsic interference. On the other hand, IIM-CBF 2 is proposed to overcome the dimensionality constraint that $M_{\mathrm{R}}^{(\mathrm{tot})}$ must not exceed $M_{\mathrm{T}}^{(\mathrm{BS})}$ in the singlecell FBMC/OQAM-based multi-user MIMO downlink. The complete precoding matrix (i.e., for the $q$ th user $\boldsymbol{F}_{q} \boldsymbol{G}_{q}$ ) that mitigates the multi-user interference and the intrinsic interference is computed iteratively and jointly with the decoding matrix. Unlike the first two schemes, IIM-CBF 3, as a novel FBMC/OQAM-based CoMP technique, is devised to enable the joint transmission of adjacent cells and to mitigate both the intra-cell interference and the inter-cell interference. In the meantime, it is able to achieve the suppression of the intrinsic interference inherent in FBMC/OQAM-based systems. Although the three IIM-CBF schemes exhibit satisfactory capabilities in alleviating the dimensionality constraint and in combating different types of interference, a complete elimination of the intrinsic interference that is inherent in FBMC/OQAM-based systems is not achieved. We revisit this issue in Section 5.2. It is worth noting that compared to the transmission strategy reviewed in Section 2.1, the residual intrinsic interference in case of the IIM-CBF schemes is much smaller, which is reflected in the performance comparison in Section 5. Throughout this work, equal power allocation is considered. A direct implementation of optimal power allocation schemes developed for CP-OFDM-based systems suffers from the loss of optimality due to the residual intrinsic interference existing in FBMC/OQAM-based multi-user MIMO downlink systems where the IIM-CBF algorithms are employed. Although the power optimization issue is beyond the scope of this paper, it is of interest to devise power allocation schemes which are tailored for FBMC/OQAMbased multi-user MIMO downlink systems by taking into account the methods of the multi-user and intrinsic interference mitigation as well as possible residual interference.

\section{Simulation results}

In this section, we evaluate the BER and the sum rate performances of the proposed IIM-CBF techniques in various simulation settings. For all examples, the number of subcarriers is 1,024 and the total bandwidth is 10 MHz. In the case of CP-OFDM, the length of the CP is set to $1 / 8$ of the symbol period. The ITU Ped-A channel or the ITU Veh-A channel [27] is adopted. Moreover, the PHYDYAS prototype filter [25] with the overlapping 
factor $K=4$ is employed. The data symbols are drawn from a 16 quadrature amplitude modulation (16 QAM) constellation. Perfect CSI is assumed at the transmitter and at the receiver.

\subsection{Single-cell multi-user MIMO downlink}

In the first experiment, a symmetric single-cell multiuser MIMO downlink setting is considered, where the base station is equipped with $M_{\mathrm{T}}^{(\mathrm{BS})}=8$ transmit antennas, each of the two users has four receive antennas, and the number of spatial streams transmitted to each user is 3. The BER performances of the three schemes for FBMC/OQAM-based systems are presented and also compared to that of a CP-OFDM-based system in Figure 2. It can be found that IIM-CBF 1 described in Section 3.1 yields the best performance. The multiuser interference is first mitigated by using the $\mathrm{BD}$ algorithm [14], and the precoding matrix and the decoding matrix are computed jointly and iteratively for each of the resulting $4 \times 4$ equivalent point-to-point transmissions. Note that the decoding matrix is initialized as (25). In this case, we observe that it takes only two iterations to converge. On the other hand, the transmission scheme that is a straightforward extension of BD [14] for the CP-OFDM case as described in Section 2.1 relies on the assumption that the channel frequency responses remain the same across adjacent subcarriers. As the ITU Veh-A channel exhibits frequency selectivity and such an assumption is therefore violated, the performance of this scheme degrades especially in the high signal-to-noise ratio (SNR) regime. We can also observe that in this

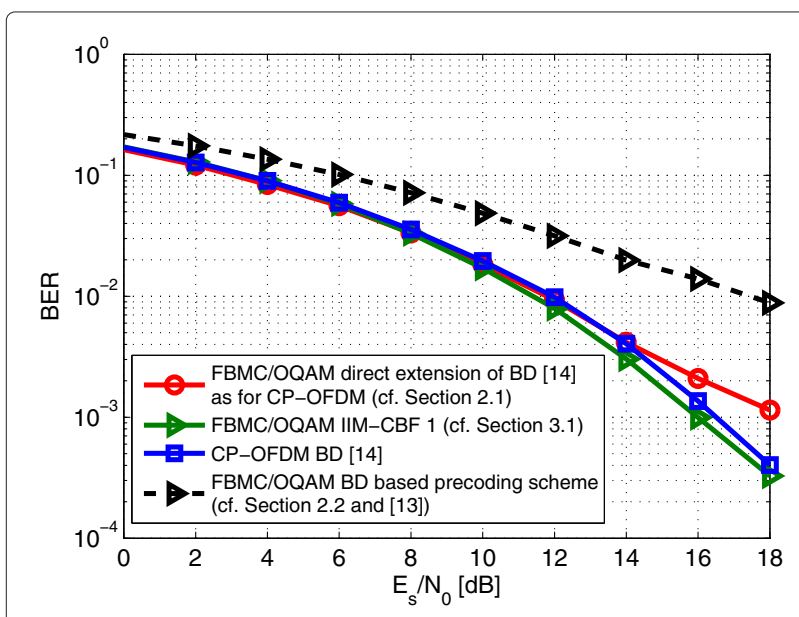

Figure 2 Comparison of the BER performances of different schemes in a symmetric multi-user MIMO downlink system. Multi-user MIMO downlink settings where $Q=2, M_{T}^{(\mathrm{BS})}=M_{\mathrm{R}}^{(\mathrm{tot})}=8$, $d=6$; the ITU Veh-A channel is considered; $E_{s}$ denotes the energy of each 16 QAM symbol, and $N_{0}$ represents the noise power spectral density. symmetric scenario, the BD-based precoding algorithm ${ }^{\mathrm{i}}$ proposed in [13] performs much worse compared to the other schemes.

In the second and the third examples, we consider single-cell multi-user MIMO downlink settings where the base station equipped with $M_{\mathrm{T}}^{(\mathrm{BS})}=8$ transmit antennas simultaneously serves two users and three users, i.e., $Q=2$ and 3, respectively. Each of the two users in the second scenario has five receive antennas, and the number of data streams transmitted to each user is 3 . On the other hand, the three users in the third scenario are equipped with three receive antennas each, and two data streams are transmitted to each of the three users. Note that for such $M_{\mathrm{R}}^{(\mathrm{tot})}>M_{\mathrm{T}}^{(\mathrm{BS})}$ configurations, the transmission strategy proposed in [13] and briefly reviewed in Section 2.2 cannot be employed. Figures 3 and 4 show the BER curves for these two scenarios, respectively. Two techniques are considered for the FBMC/OQAM-based system, i.e., IIM-CBF 2 presented in Section 3.2 and a direct extension ( $c f$. Section 2.1) of the LoCCoBF algorithm [24] originally designed for the case of CP-OFDM. For the purpose of comparison, we also present the BER performance of a CP-OFDM-based system with the same configuration where LoCCoBF is employed. For both the proposed IIM-CBF 2 scheme and LoCCoBF, $\epsilon$ for the stopping criterion is set to $10^{-5}$, and the maximum number of iterations ${ }^{j}$ is 50 . It can be observed that the performance of the FBMC/OQAM-based multi-user MIMO downlink system where IIM-CBF 2 is employed is slightly better than its CP-OFDM-based counterpart due to the fact that no insertion of the $\mathrm{CP}$ is required. The other transmission scheme for the FBMC/OQAM-based system

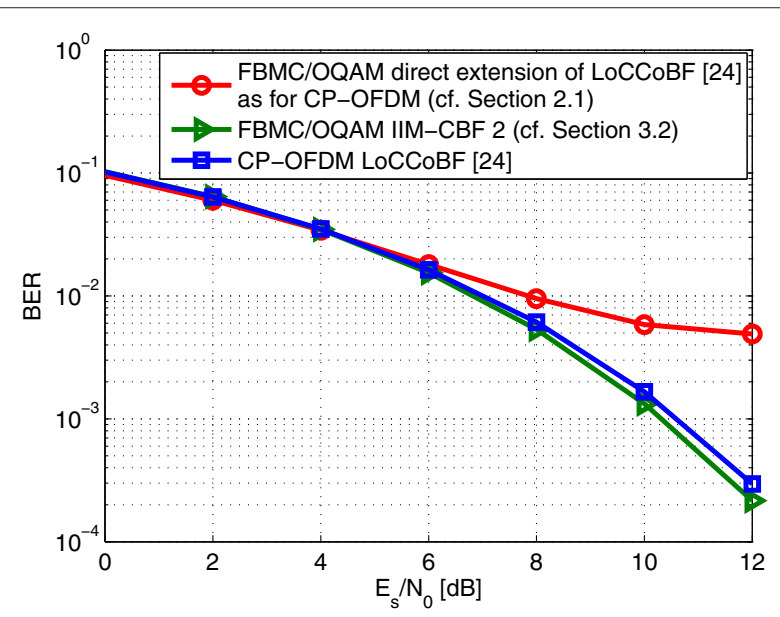

Figure 3 Comparison of the BER performances of different schemes in a multi-user MIMO downlink system. Multi-user MIMO downlink settings where $Q=2, M_{T}^{(\mathrm{BS})}=8, M_{R}^{(\text {tot })}=10, d=6$; the ITU Ped-A channel is considered; $E_{\mathrm{S}}$ denotes the energy of each $16 \mathrm{QAM}$ symbol, and $N_{0}$ represents the noise power spectral density. 


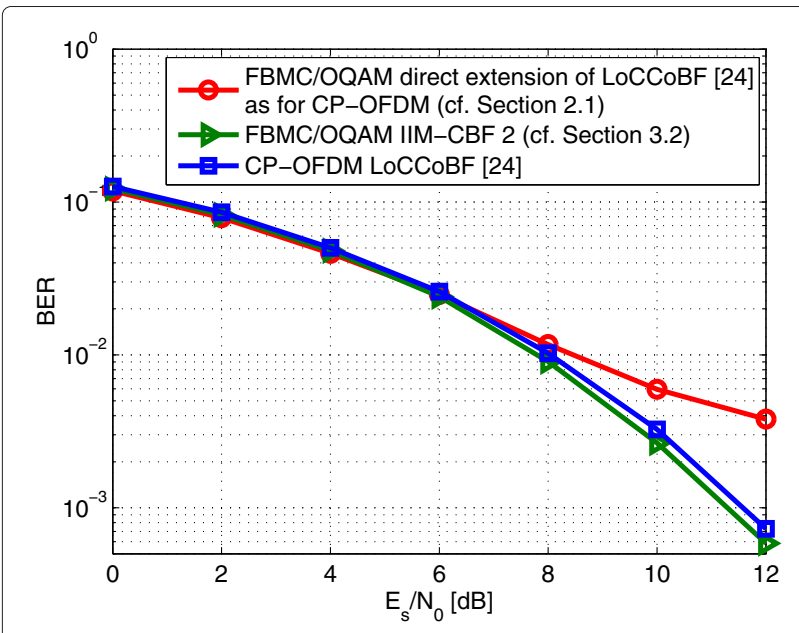

Figure 4 Comparison of the BER performances of different schemes in a multi-user MIMO downlink system. Multi-user MIMO downlink settings where $Q=3, M_{T}^{(\mathrm{BS})}=8, M_{\mathrm{R}}^{(\mathrm{tot})}=9, d=6$; the ITU Ped-A channel is considered; $E_{s}$ denotes the energy of each 16 QAM symbol, and $N_{0}$ represents the noise power spectral density.

suffers from a performance degradation due to the frequency selectivity of the channel. By assuming that the channel stays constant across the neighboring subcarriers, the multi-user interference and the intrinsic interference cannot be eliminated even for high SNRs.

In addition, the convergence behavior of IIM-CBF 2 for the single-cell multi-user MIMO FBMC/OQAM downlink system where $M_{\mathrm{R}}^{(\mathrm{tot})}>M_{\mathrm{T}}^{(\mathrm{BS})}$ is investigated. The complimentary cumulative distribution function (CCDF) of the number of iterations required to achieve the convergence of the IIM-CBF 2 scheme presented in Section 3.2 and LoCCoBF for the case of CP-OFDM is illustrated in Figure 5. The three-user scenario used for Figure 4 is considered. By comparison, we also plot the same set of results for a four-user case, i.e., $Q=4$. The base station has eight transmit antennas, and each of the four users is equipped with three receive antennas and is sent to two data streams. Therefore, the total number of the receive antennas of the users $M_{\mathrm{R}}^{(\text {tot })}=12$ and all spatial degrees of freedom are exploited.

It can be observed that for the three-user scenario, IIM-CBF 2 converges within six iterations in almost all of the cases. As the number of users and consequently the total number of receive antennas increase, the number of iterations needed for the convergence becomes slightly larger. Nevertheless, the convergence is achieved within ten iterations. Moreover, we can see from the comparison of the proposed IIM-CBF 2 technique for the FBMC/OQAM-based system and LoCCoBF for the case of CP-OFDM that the number of iterations required for the convergence for both schemes is similar. Hence, compared to the CP-OFDM-based multi-user MIMO

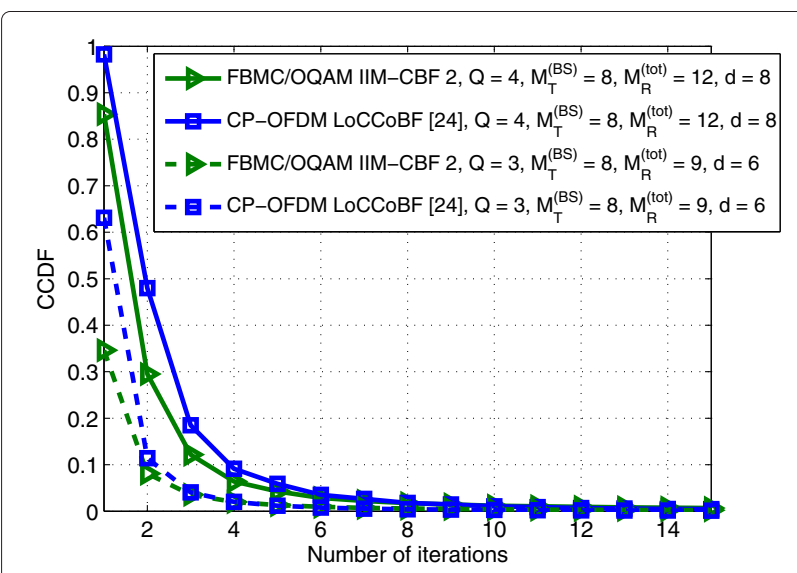

Figure 5 CCDF of the number of iterations required for IIM-CBF 2 and LoCCoBF. CCDF of the number of iterations required for IIM-CBF 2 and LoCCOBF in multi-user MIMO FBMC/OQAM and CP-OFDMbased downlink systems with different settings, respectively; the ITU Ped-A channel is considered.

downlink setting, employing such an IIM-CBF technique in the FBMC/OQAM-based system where $M_{\mathrm{R}}^{(\text {tot })}>M_{\mathrm{T}}^{(\mathrm{BS})}$ does not result in an increased number of iterations for the convergence. Only the processing dedicated to the elimination of the intrinsic interference contributes to a slight additional complexity.

Furthermore, we consider again the four-user single-cell multi-user MIMO downlink system (as used in Figure 5) and investigate the impact of the residual CFO. The IIMCBF 2 scheme for the FBMC/OQAM-based system and LoCCoBF for CP-OFDM are considered. In addition, it is assumed that the residual CFO (normalized by the subcarrier spacing) ranges ${ }^{\mathrm{k}}$ either from 0 to 0.1 or from 0 to 0.15 . Figure 6 illustrates the corresponding BER performances of these two schemes in the presence of the residual CFO. Notice that the CP-OFDM-based system suffers from a severe performance degradation. In contrast, the FBMC/OQAM-based system with IIM-CBF 2 significantly outperforms its CP-OFDM-based counterpart, and it exhibits a greater robustness against the synchronization errors in the frequency domain.

\subsection{CoMP downlink}

In the sequel, the performance of the FBMC/OQAMbased CoMP downlink is assessed. Instead of BER, we choose to use the sum rate performance as the evaluation approach. We start with a two-cell scenario consisting of five users in total. Each cell equipped with four transmit antennas serves three users each with two receive antennas, which forms a $4 \times 6$ multi-user downlink setting. Among the three users, two are cell interior users, and one is a cell edge user. A single data stream is transmitted to each cell interior user, and two data streams 


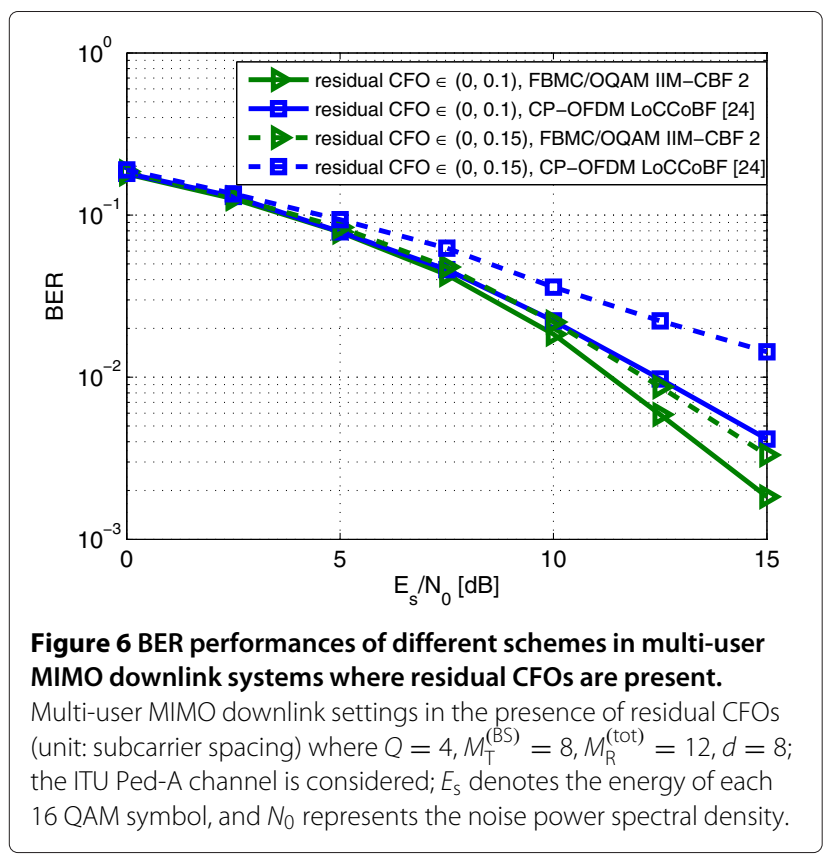

are transmitted to the cell edge use, i.e., for each cell full spatial multiplexing is considered. The path loss of the transmission to the cell edge users is assumed to be ten times bigger than that for the cell interior users [19]. To calculate the sum rate for both the FBMC/OQAM and the CP-OFDM cases, we first compute an instantaneous signal-to-interference-plus-noise-ratio (SINR) on each data stream for each subcarrier. In case of the FBMC/OQAM-based multi-user MIMO downlink where IIM-CBF 2 is employed, the decoded signal is written as

$$
\begin{aligned}
\hat{\boldsymbol{d}}_{k}[n]= & \boldsymbol{D}_{k}^{\mathrm{T}}[n] \cdot \operatorname{Re}\left\{\boldsymbol{H}_{k}[n] \boldsymbol{F}_{k}[n] \boldsymbol{d}_{k}[n]\right. \\
& \left.+\sum_{i=n-3}^{n+3} \sum_{\ell=k-1}^{k+1} \boldsymbol{H}_{\ell}[i] \boldsymbol{F}_{\ell}[i] c_{i \ell} \boldsymbol{d}_{\ell}[i]+\boldsymbol{n}_{k}[n]\right\} \\
= & \boldsymbol{D}_{k}^{\mathrm{T}}[n] \cdot \operatorname{Re}\left\{\boldsymbol{H}_{k}[n] \boldsymbol{F}_{k}[n]\right\} \boldsymbol{d}_{k}[n] \\
& +\boldsymbol{D}_{k}^{\mathrm{T}}[n] \cdot \operatorname{Im}\left\{-\sum_{i=n-3}^{n+3} \sum_{\ell=k-1}^{k+1} \boldsymbol{H}_{\ell}[i] \boldsymbol{F}_{\ell}[i]\right\} \bar{c}_{i \ell} \overline{\boldsymbol{d}}_{\ell}[i] \\
& +\boldsymbol{D}_{k}^{\mathrm{T}}[n] \cdot \operatorname{Re}\left\{\boldsymbol{n}_{k}[n]\right\}, \quad(\ell, i) \neq(k, n) .
\end{aligned}
$$

where

$$
\begin{aligned}
& \bar{c}_{i \ell}=\operatorname{Im}\left\{c_{i \ell}\right\}, \quad \overline{\boldsymbol{d}}_{\ell}[i]=\boldsymbol{d}_{\ell}[i], \quad \text { when } \quad(\ell+i) \text { is even, } \\
& \bar{c}_{i \ell}=c_{i \ell}, \quad \overline{\boldsymbol{d}}_{\ell}[i]=\operatorname{Im}\left\{\boldsymbol{d}_{\ell}[i]\right\}, \quad \text { when } \quad(\ell+i) \quad \text { is odd. }
\end{aligned}
$$

Note that as mentioned in Section 2, for the case where $(\ell+i)$ is even, the signal $\boldsymbol{d}_{\ell}[i]$ on the $\ell$ th subcarrier and at the $i$ th time instant is real, while the corresponding coefficient $c_{i \ell}$ is pure imaginary. On the contrary, when $(\ell+i)$ is odd, $\boldsymbol{d}_{\ell}[i]$ and $c_{i \ell}$ are pure imaginary and real, respectively. This explains the definitions of $\bar{c}_{i \ell}$ and $\overline{\boldsymbol{d}}_{\ell}[i]$ shown above. Let us further write (43) as

$$
\begin{aligned}
\hat{\boldsymbol{d}}_{k}[n]= & \boldsymbol{H}_{k}^{(\mathrm{eff})}[n] \boldsymbol{d}_{k}[n]+\sum_{i=n-3}^{n+3} \sum_{\ell=k-1}^{k+1} \\
& \times \boldsymbol{H}_{\ell}^{(\mathrm{inf})}[i] \overline{\boldsymbol{d}}_{\ell}[i]+\widetilde{\boldsymbol{n}}_{k}[n], \quad(\ell, i) \neq(k, n),
\end{aligned}
$$

where

$$
\begin{aligned}
\boldsymbol{H}_{k}^{(\mathrm{eff})}[n] & =\boldsymbol{D}_{k}^{\mathrm{T}}[n] \cdot \operatorname{Re}\left\{\boldsymbol{H}_{k}[n] \boldsymbol{F}_{k}[n]\right\} \in \mathbb{R}^{d \times d}, \\
\boldsymbol{H}_{\ell}^{(\mathrm{inf})}[i] & =\boldsymbol{D}_{k}^{\mathrm{T}}[n] \cdot \operatorname{Im}\left\{-\boldsymbol{H}_{\ell}[i] \boldsymbol{F}_{\ell}[i]\right\} \bar{c}_{i \ell} \in \mathbb{R}^{d \times d}, \\
\widetilde{\boldsymbol{n}}_{k}[n] & =\boldsymbol{D}_{k}^{\mathrm{T}}[n] \cdot \operatorname{Re}\left\{\boldsymbol{n}_{k}[n]\right\} .
\end{aligned}
$$

To calculate the SINR denoted by $\gamma_{r, k}$ for the $r$ th data stream on the $k$ th subcarrier, where $r=1, \ldots, d$, the entry $(r, r)$ of $\boldsymbol{H}_{k}^{(\mathrm{eff})}[n]$ determines the effective gain for the $r$ th data stream, while the entries $(r, u)(u=1, \ldots, d, u \neq r)$ of $\boldsymbol{H}_{k}^{\text {(eff) }}[n]$ determine the residual multi-user and interstream interference. In addition, the elements of the $r$ th row of $\boldsymbol{H}_{\ell}^{(\mathrm{inf})}[i]$ provide a measure of the residual intrinsic interference. Notice that only when $\boldsymbol{D}_{k}[n]=\boldsymbol{D}_{\ell}[i](\ell=$ $k-1, k, k+1, i=n-3, \ldots, n+3$, and $(\ell, i) \neq(k, n))$, $\boldsymbol{H}_{\ell}^{(\text {inf) }}[i]$ is rendered as zero, and the intrinsic interference is completely eliminated. Due to the fact that the channel is not flat fading, the aforementioned condition is not fulfilled, leading to the existence of the residual intrinsic interference. The noise component can be extracted from the covariance matrix of $\widetilde{\boldsymbol{n}}_{k}[n]$

$$
\boldsymbol{R}_{\widetilde{n} \widetilde{n}_{k}}[n]=\boldsymbol{D}_{k}^{\mathrm{T}}[n] \cdot \frac{N_{0}}{2} \boldsymbol{I}_{M_{\mathrm{R}}} \cdot \boldsymbol{D}_{k}[n]
$$

Therefore, $\gamma_{r, k}$ is computed as

$$
\gamma_{r, k}=\frac{\left(\boldsymbol{H}_{k}^{\text {(eff) }}[n](r, r)\right)^{2}}{\sum_{u=1, u \neq r}^{d}\left(\boldsymbol{H}_{k}^{(\mathrm{eff})}[n](r, u)\right)^{2}+\sum_{\substack{i=n-3 \\(\ell, i) \neq(k, n)}}^{n+3} \sum_{\substack{\ell=k-1 \\ k+1}}^{d}\left(\boldsymbol{H}_{\ell}^{\text {(inf) }}[i](r, u)\right)^{2}+\left(\boldsymbol{R}_{\widetilde{n}_{n}}[n](r, r)\right)^{2}} .
$$


The sum rate is then approximated as

$$
R_{\text {sum }}=\frac{1}{N_{\mathrm{c}}} \sum_{k=1}^{N_{\mathrm{c}}} \sum_{r=1}^{d} \log _{2}\left(1+\gamma_{r, k}\right),
$$

where $N_{\mathrm{c}}$ is the total number of subcarriers. For the case of CP-OFDM, the sum rate can be computed similarly, but the residual intrinsic interference term does not exist. Moreover, the $\operatorname{Re}\{\cdot\}$ operation is not used. On the other hand, when a transmission scheme that is a straightforward extension of the CP-OFDM case ( $c f$. Section 2.1) is adopted, the residual intrinsic interference results from the violation of the assumption that the channels of adjacent subcarriers are the same. Therefore, the effective channel that determines the intrinsic interference caused by the signal on the $\ell$ th subcarrier and at the $i$ th time instant to that on the $k$ th subcarrier and at the $n$th time instant, where $(\ell, i) \neq(k, n)$, is expressed as

$$
\boldsymbol{H}_{\ell}^{(\mathrm{inf})}[i]=\operatorname{Im}\left\{\boldsymbol{D}_{k}^{\mathrm{H}}[n] \boldsymbol{H}_{\ell}[i] \boldsymbol{F}_{\ell}[i]-\boldsymbol{D}_{k}^{\mathrm{H}}[n] \boldsymbol{H}_{k}[n] \boldsymbol{F}_{k}[n]\right\} \bar{c}_{i \ell} .
$$

Then, the sum rate for this case can be calculated accordingly. It is worth mentioning that in case of the downlink of the CoMP system, the fact that adjacent cells send the same signal to each cell edge user should be taken into account in the sum rate calculation, while the rest resembles the aforementioned case of the multi-user MIMO downlink system.

To this end, we use Figure 7 to illustrate the sum rate performances of two transmission schemes for the FBMC/OQAM-based CoMP downlink systems described at the beginning of this section. Here $\mathrm{SNR}=P_{\mathrm{T}} / \sigma_{n}^{2}$, where $P_{\mathrm{T}}$ is the transmit power of each cell. The
CP-OFDM-based system where Extended FlexCoBF ${ }^{1}$ is employed is also considered for comparison. It can be seen that IIM-CBF 3 leads to a slightly better performance of the FBMC/OQAM-based system in contrast to the CPOFDM-based system. The reason is that the former has a higher spectral efficiency compared to the latter, since no CP is required. This observation also implies the effectiveness of the proposed transmission strategy IIM-CBF 3 in mitigating the intra-cell and inter-cell interference as well as the intrinsic interference. By comparison, when a transmission strategy originally designed for CP-OFDM, here Extended FlexCoBF, is straightforwardly extended to the FBMC/OQAM system and the channel is frequency selective, the performance is much worse than that of the proposed IIM-CBF 3 scheme due to a much higher level of the residual interference.

Moreover, Figure 8 depicts the CCDF of the number of iterations required for the IIM-CBF 3 technique to converge. For all the evaluated schemes, $\epsilon$ used for the stopping criterion is set to $10^{-5}$, and the iterative procedure is manually terminated when the number of iterations reaches 50 . Two types of the frequency selective channel, ITU Ped-A and Veh-A, are considered. Recall that in the initialization step of IIM-CBF 3, the decoding matrices calculated for the previous subcarrier are used to initialize the decoding matrices for the current subcarrier. Therefore, the lower the frequency selectivity of the channel is, the more effective becomes such a choice of the initial decoding matrices, which leads to a smaller number of iterations required for the convergence of the coordinated beamforming algorithm. Consequently, we observe in Figure 8 that compared to the case of the ITU Veh-A channel which is more frequency selective, the number of

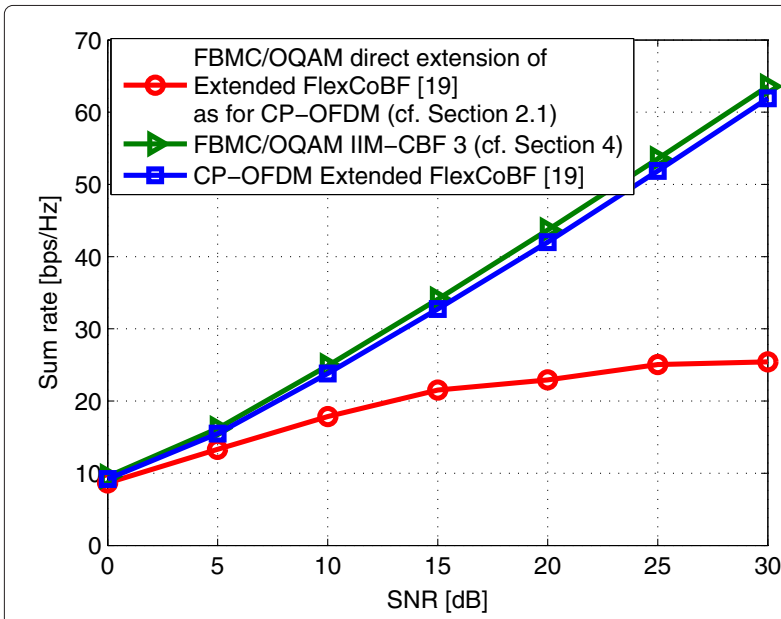

Figure 7 Comparison of the sum rate performances of different coordinated beamforming schemes in the CoMP downlink. CoMP downlink settings where the total number of users in two adjacent cells is 5; the ITU Ped-A channel is considered.

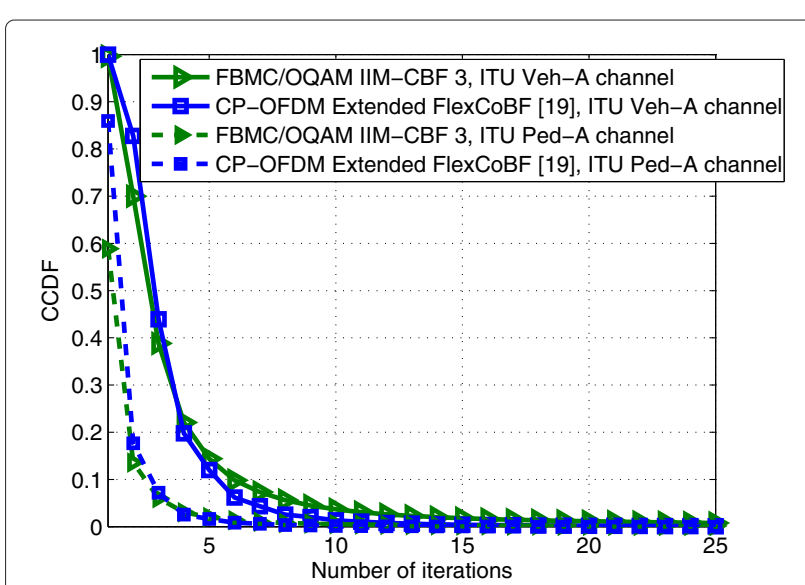

Figure 8 CCDF of the number of iterations required for IIM-CBF 3 and Extended FlexCoBF. CoMP downlink settings where the total number of users in two adjacent cells is 5; the ITU Ped-A channel or the ITU Veh-A channel is considered. 
iterations that are needed by the coordinated beamforming schemes to converge in case of the ITU Ped-A channel is slightly smaller. However, even when the ITU Veh-A is considered as the propagation channel, the proposed IIMCBF 3 technique converges within 15 iterations in almost all of the cases.

Finally, a three-cell CoMP downlink scenario is considered. Each cell equipped with four transmit antennas serves three users each with two receive antennas. This leads to a $4 \times 6$ multi-user downlink setting. Among the three users, two are cell interior users, and one is a cell edge user. A single data stream is transmitted to each cell interior user, and two data streams are transmitted to the cell edge user. The numerical results with respect to the sum rate performance are shown in Figure 9. Similar observations are obtained as in Figure 7.

\section{Conclusions}

We have developed three IIM-CBF based transmission schemes for the downlink of FBMC/OQAM based multiuser MIMO systems and CoMP systems. The first algorithm that is called IIM-CBF 1 is designed for a multi-user MIMO FBMC/OQAM downlink system where $M_{\mathrm{T}}^{(\mathrm{BS})}=$ $M_{\mathrm{R}}^{\text {(tot) }}$. We have employed an iterative procedure to jointly compute the precoding matrix and the decoding matrix of each equivalent single-user transmission that results from the elimination of the multi-user interference. On the other hand, the IIM-CBF 2 technique has been proposed as a solution to the problem that the state-of-the-art transmission strategies for the downlink of FBMC/OQAM-based multi-user MIMO systems fail to work when $M_{\mathrm{R}}^{(\mathrm{tot})}>M_{\mathrm{T}}^{(\mathrm{BS})}$. Moreover, we have conducted

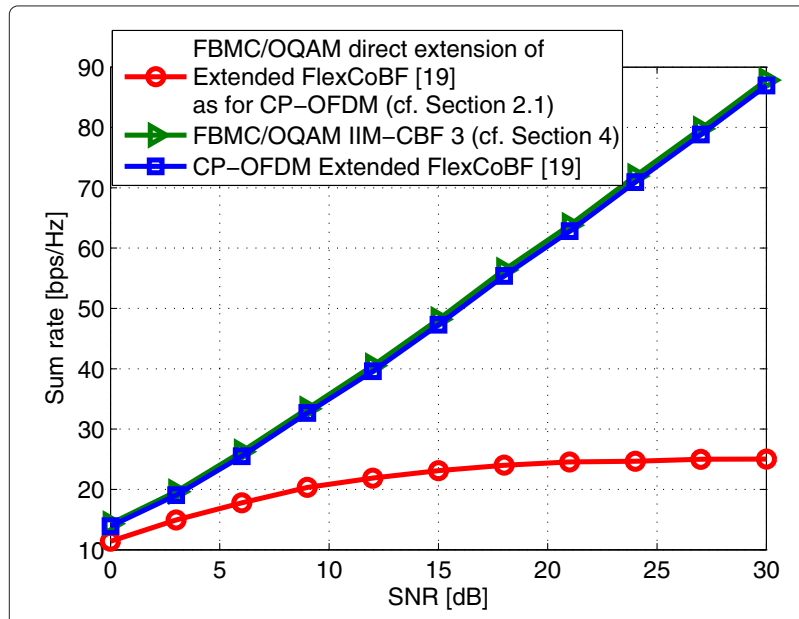

Figure 9 Comparison of the sum rate performances of different coordinated beamforming schemes in the CoMP downlink. CoMP downlink settings where the total number of users in three adjacent cells is 7; the ITU Ped-A channel is considered. an investigation of FBMC/OQAM-based CoMP downlink systems for the first time. With a focus on the case of partial cooperation of adjacent cells, the scheme, IIM-CBF 3 , has been designed to enable the joint transmission of base stations in adjacent cells and combat both the intracell and the inter-cell interference. It is worth noting that in addition to the suppression of the multi-user interference, these three proposed IIM-CBF schemes are effective in mitigating the intrinsic interference that is inherent in FBMC/OQAM-based systems without assuming that the propagation channel is almost flat fading. To demonstrate the advantages of the three IIM-CBF algorithms, their BER and sum rate performances have been shown in different settings. Via the simulation results, it has been shown that the FBMC/OQAM-based multi-user MIMO and CoMP downlink systems where IIM-CBF 1, IIM-CBF 2 , or IIM-CBF 3 is employed achieve a similar performance compared to their CP-OFDM-based counterparts but with a higher spectral efficiency and a greater robustness against misalignments in the frequency domain. In addition, we have numerically analyzed the convergence of the IIM-CBF techniques. It leads to the conclusion that the additional complexity is quite acceptable as the price of alleviating the aforementioned dimensionality constraint.

\section{Endnotes}

${ }^{\text {a }}$ Parts of this paper have been published in ICASSP 2014 [28] and ISCCSP 2014 [29].

${ }^{\mathrm{b}}$ Here we only provide the formulas of the channel matrices, precoding matrices, and data vectors on the $k$ th subcarrier and at the $n$th time instant explicitly due to limited space. In case of the $\ell$ th subcarrier and the $i$ th time instant, the corresponding expressions can be obtained by replacing $k$ and $n$ with $\ell$ and $i$, respectively.

${ }^{\mathrm{c}}$ For the case where $(k+n)$ is odd, the desired signal on the $k$ th subcarrier and at the $n$th time instant is pure imaginary, while the intrinsic interference is real providing that the prototype pulse satisfies the perfect reconstruction property $[7,12]$. As the two cases are essentially equivalent to each other, we only take the case where $(k+n)$ is even to describe the proposed algorithm in this paper. In addition, each entry in the data vector corresponds to either the in-phase component or the quadrature component of a QAM symbol that is assumed to have unit energy.

'In IIM-CBF 3 for the CoMP downlink, $\boldsymbol{F}_{q}$ suppresses both the intra-cell interference and the inter-cell interference.

${ }^{\mathrm{e}}$ Other receivers, such as zero forcing or maximal ratio combining, can also be employed in this coordinated beamforming algorithm.

${ }^{\mathrm{f}}$ These two stopping criteria require different thresholds for the convergence. In this work, we only 
present numerical results obtained when the stopping criterion as in (34) is employed, and the threshold $\epsilon$ is set to $10^{-5}$.

In this example, we assume that the indices of each cell edge user in Cell 1 and Cell 2 are the same to facilitate the description of the scenario.

${ }^{\mathrm{h}}$ In such scenarios where multiple data streams are transmitted to at least one user, by employing the MMSE or the MRC to update the decoding matrices in IIM-CBF 3 , convergence cannot be achieved.

${ }^{\mathrm{i}}$ In the implementation of this algorithm, after the cancelation of the multi-user interference by using BD, only the ZF based step that ensures the cancelation of the intrinsic interference is considered. The remaining part of the transmit processing (spatial mapping) and the receive processing (MMSE receiver) are chosen to be the same as the other schemes for the purpose of a fair comparison. Note that the precoding algorithm proposed in [13] is dominated by the BD and ZF based steps.

jVia numerical simulations, it has been observed that there exist rare cases where it takes a large number of iterations for the stopping criteria of the IIM-CBF schemes to be fulfilled. Therefore, a maximum number of iterations is set to handle these cases. The iterative procedure is manually terminated if the stopping criteria are not fulfilled when the number of iterations reaches this maximum number. Taking IIM-CBF 2 as an example, its stopping criterion corresponds to the residual interference. We have observed that when the algorithm is manually terminated, though the residual interference is above the threshold, its value is already so small that the performance is not affected much. Except for this simple way of setting a maximum number of iterations, the termination of the iterative procedure can also be determined based on the variation of the residual interference (in case of IIM-CBF 2 and IIM-CBF 3) or the change of the precoding matrix (in case of IIM-CBF 1) as the number of iterations increases.

${ }^{\mathrm{k}}$ In the simulations, the residual CFO is drawn uniformly from the range $(0,0.1)$ or $(0,0.15)$.

${ }^{1}$ In the implementation of Extended FlexCoBF [19], we adopt the same mechanism of initializing the decoding matrices as in the LoCCoBF algorithm [24] such that the correlation of the channels of adjacent subcarriers is exploited, and consequently the number of iterations required for the convergence is reduced.

\section{Competing interests}

The authors declare that they have no competing interests.

\section{Acknowledgements}

The authors gratefully acknowledge the financial support by the European Union FP7-ICT project EMPhAtiC (http://www.ict-emphatic.eu) under grant agreement no. 318362 .

Received: 6 December 2013 Accepted: 19 May 2014

Published: 9 June 2014

\section{References}

1. MG Bellanger, Specification and design of a prototype filter for filter bank based multicarrier transmission, in Proceedings of the IEEE International Conference on Acoustics, Speech, and Signal Processing (Salt Lake City, May 2001)

2. P Siohan, C Siclet, N Lacaille, Analysis and design of OFDM/OQAM systems based on filterbank theory. IEEE Trans. Signal Process. 50(5), 1170-1183 (2002)

3. T Fusco, A Petrella, M Tanda, Sensitivity of multi-user filter-bank multicarrier systems to synchronization errors, in Proceedings of ISCCSP (St Julians, March 2008)

4. H Saeedi-Sourck, Y Wu, JWM Bergmans, S Sadri, B Farhang-Boroujeny, Complexity and performance comparison of filter bank multicarrier and OFDM in uplink of multicarrier multiple access networks. IEEE Trans. Signal Process. 59(4), 1907-1912 (2011)

5. M Shaat, F Bader, Computationally efficient power allocation algorithm in multicarrier-based cognitive radio networks: OFDM and FBMC systems. EURASIP J. Adv. Signal Process. 2010 (2010)

6. M Shaat, F Bader, Comparison of OFDM and FBMC performance in multi-relay cognitive radio network, in Proceedings of 9-th International Symposium on Wireless Communications Systems (ISWCS 2012) (Paris, August 2012)

7. MG Bellanger, FBMC physical layer: a primer (June 2010)

8. R Zakaria, D le Ruyet, M Bellanger, Maximum Likelihood Detection in spatial multiplexing with FBMC, in Proceedings of the 2010 European Wireless Conference (Lucca, June 2010)

9. Y Cheng, M Haardt, Widely linear processing in MIMO FBMC/OQAM systems, in Proceedings of the 10-th International Symposium on Wireless Communications Systems (ISWCS 2013) (IImenau, August 2013)

10. M Caus, Al Perez-Neira, Multi-stream transmission in MIMO-FBMC systems, in Proceedings of the IEEE International Conference on Acoustics, Speech, and Signal Processing (ICASSP) (Vancouver, May 2013)

11. M Caus, Al Perez-Neira, Comparison of linear and widely linear processing in MIMO-FBMC systems, in Proceedings of the 10-th International Symposium on Wireless Communications Systems (ISWCS 2013) (IImenau, August 2013)

12. M Caus, Al Perez-Neira, SDMA for filterbank with Tomlinson Harashima precoding, in Proceedings of ICC 2013, Budapest, June, (2013)

13. M Caus, Perez-Neira Al, Moretti M, SDMA for FBMC with block diagonalization, in Proceedings of SPAWC 2013 (Darmstadt, June 2013)

14. QH Spencer, AL Swindlehurst, M Haardt, Zero-forcing methods for downlink spatial multiplexing in multi-user MIMO channels. IEEE Trans. Signal Process. 52(2), 461-471 (2004)

15. F Horlin, J Fickers, T Deleu, J Louveaux, Interference-free SDMA for FBMC-OQAM. EURASIP J. Adv. Signal Process. 2013 (2013)

16. M Newinger, LG Baltar, AL Swindlehurst, JA Nossek, MISO Broadcasting FBMC system for highly frequency selective channels, in Proceedings of the International ITG Workshop on Smart Antennas (WSA 2014) (Erlangen, March 2014)

17. K Kim, J Lee, C Lee, N Jeon, S Kim, Coordinated beamforming with limited BS cooperation for multicell multiuser MIMO broadcast channel, in Proceedings of the IEEE Vehicular Technology Conference (VTC) Spring (Barcelona, April 2009)

18. D Lee, H Seo, B Clerckx, E Hardouin, D Mazzarese, S Nagata, K Sayana, Coordinated multipoint transmission and reception in LTE-advanced deployment scenarios and operational challenges. IEEE Commun. Mag. 50(2), 148-155 (2012)

19. B Song, F Roemer, M Haardt, Flexible coordinated beamforming (FlexCoBF) for the downlink of multi-user MIMO systems in single and clustered multiple cells. Elsevier Signal Process. 93, 2462-2473 (2013)

20. L Venturino, $N$ Prasad, $X$ Wang, Coordinated linear beamforming in downlink multi-cell wireless networks. IEEE Trans. Wireless Commun. 9(4), $1536-1276$ (2010)

21. J Zhang, R Chen, JG Andrews, Networked MIMO with clustered linear precoding. IEEE Trans. Wireless Commun. 8(4), 1910-1921 (2009)

22. F Boccardim, H Huang, Limited downlink network coordination in cellular network, in Proceedings of the IEEE International Symposium on Personal Indoor and Mobile Radio Communications (Athens, September 2007)

23. H Zhang, NB Mehta, AF Molisch, J Zhang, H Dai, Asynchronous interference mitigation in cooperative base station systems. IEEE Trans. Wireless Commun. 7(1), 155-164 (2008) 
24. Y Cheng, S Li, J Zhang, F Roemer, B Song, M Haardt, Y Zhou, M Dong, An efficient and flexible transmission strategy for the multi-carrier multi-user MIMO downlink. IEEE Trans. Vehicular Technol. 63(2), 628-642 (2014)

25. FP7-ICT Project PHYDYAS - Physical Layer for Dynamic Spectrum Access and Cognitive Radio. http://www.ict-phydyas.org.

26. V Stankovic, M Haardt, Generalized design of multi-user MIMO precoding matrices. IEEE Trans. Wireless Commun. 7(3), 953-961 (2008)

27. ITU-R Recommendation M.1225, Guidelines for evaluation of radio transmission technologies for IMT-2000 (1997)

28. Y Cheng, P Li, M Haardt, Coordinated beamforming in MIMO FBMC/OQAM systems, in Proceedings of the IEEE International Conference on Acoustics, Speech, and Signal Processing (ICASSP) (Florence, May 2014)

29. Y Cheng, P Li, M Haardt, Coordinated beamforming for the multi-user MIMO downlink using FBMC/OQAM, in Proceedings of the 6th International Symposium on Communications, Control, and Signal Processing (ISCCSP 2014) (Athens, May 2014)

\section{Submit your manuscript to a SpringerOpen ${ }^{\circ}$ journal and benefit from:}

- Convenient online submission

- Rigorous peer review

- Immediate publication on acceptance

- Open access: articles freely available online

- High visibility within the field

- Retaining the copyright to your article 